\title{
A FITNESS ANALYSIS SYSTEM WITH AN INTELLIGENT INTERFACE
}

Thesis submitted by

ALFIO V PARISI BSC(HONS) KAIP

in fulfilment of the requirements of

MASTER OF APPLIED SCIENCE

University of Central Queensland

Department of Mathematics and computing

School of Applied Science

November, 1991 
One of the problems in the physiological assessment of an athlete is poor communication of the results of the physiological tests to the coach. This thesis describes the development of an expert system, EXFIT designed to bridge the gap between the scientist and the coach and facilitate the provision of scientific information in a systematic and coherent fashion in a report to the coach. The expert system provides generalized recommendations which the coach utilizes in developing specific training schedules suited to the particular athlete to enhance performance. The recommendations are based on a series of physiological analyses of the athlete.

The analyses available in EXFIT are: anaerobic power (peak and total), aerobic power, onset of blood lactate accumulation, maximum blood lactate, blood, muscle structure (fibre type), metabolic status of muscle - aerobic capacity and anaerobic capacity. For two of these analyses, namely the anaerobic power (peak and total) and the aerobic power, provision has been made to allow for both manual and automatic acquisition of the data from the tests for which the data acquisition system was developed as part of this project. The results of the test equipment not presently interfaced to the computer are collected and entered manually.

The implementation of the expert system is described with emphasis placed on recognition of the internal structure of the knowledge, independence from a particular expert system shell and the design for future expansion and maintenance. EXFIT has been split into the four separate modules of user interface, data, information and knowledge. The data and information have been normalized and stored using the knowledge dictionary concept. The design for maintenance was tested by writing a program that allows the domain expert to modify or add to the data and information of EXFIT without the requirement for a knowledge engineer. 


\section{TABLE OF CONTENTS}

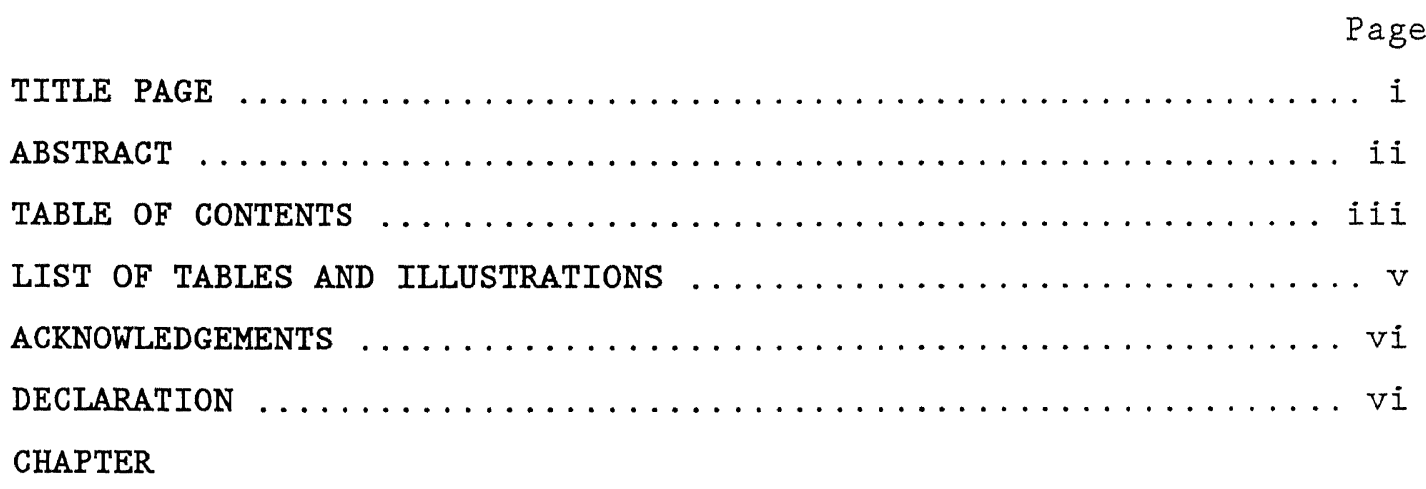

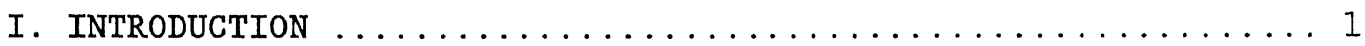

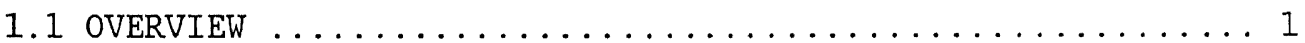

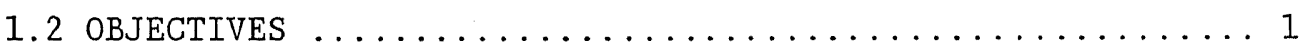

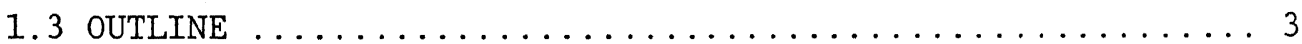

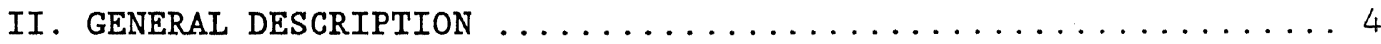

III. SURVEY OF RELEVANT RESEARCH $\ldots \ldots \ldots \ldots \ldots \ldots \ldots \ldots \ldots \ldots$

IV. DATA ACQUISITION $\ldots \ldots \ldots \ldots \ldots \ldots \ldots \ldots \ldots \ldots \ldots \ldots \ldots \ldots \ldots \ldots \ldots$

4.1 INTRODUCTION ........................... 9

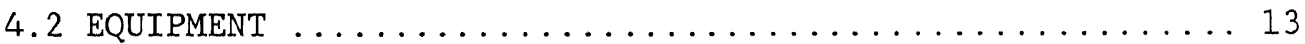

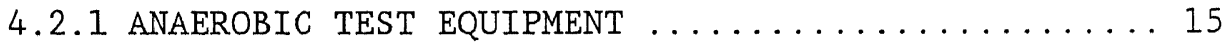

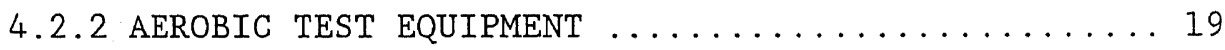

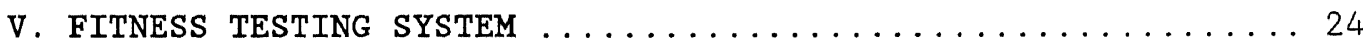

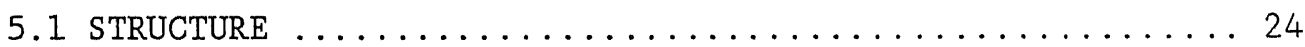

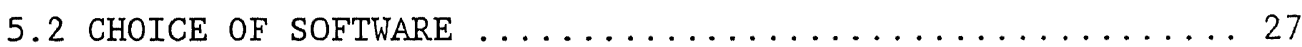

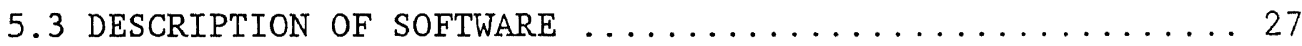

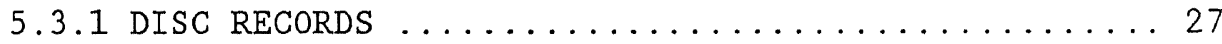

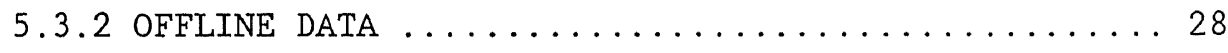

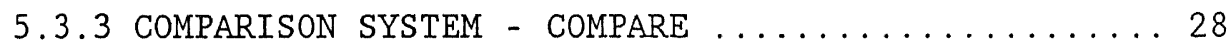

5.3.3.1 PROFILING SYSTEM ............... 29

5.3 .3 .2 PERFORMANCE SYSTEM $\ldots \ldots \ldots \ldots \ldots \ldots \ldots \ldots$

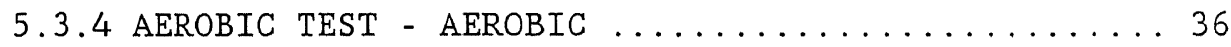

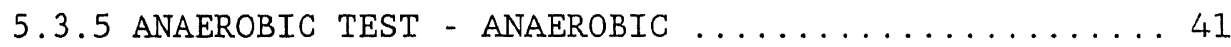

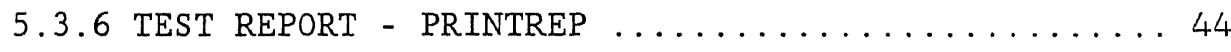

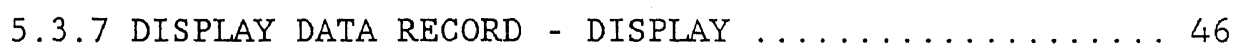

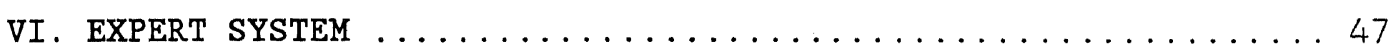

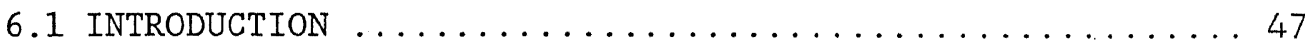


6.2 DESIGN OF THE ADVISORY SYSTEM $\ldots \ldots \ldots \ldots \ldots \ldots \ldots \ldots \ldots$

6.2 .1 OVERALL STRUCTURE $\ldots \ldots \ldots \ldots \ldots \ldots \ldots \ldots \ldots \ldots \ldots$

6.2.2 KNOWLEDGE BASE - DATA, INFORMATION AND KNOWLEDGE . 51

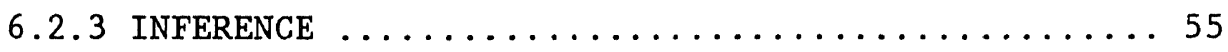

6.2 .4 RECOMMENDATIONS REPORT $\ldots \ldots \ldots \ldots \ldots \ldots \ldots \ldots \ldots$

6.3 CHOICE OF SOFTWARE PLATFORM $\ldots \ldots \ldots \ldots \ldots \ldots \ldots \ldots \ldots \ldots \ldots \ldots$

VII. EXPERT SYSTEM MAINTENANCE $\ldots \ldots \ldots \ldots \ldots \ldots \ldots \ldots \ldots \ldots \ldots \ldots \ldots \ldots \ldots \ldots$

7.1 INTRODUCTION $\ldots \ldots \ldots \ldots \ldots \ldots \ldots \ldots \ldots \ldots \ldots \ldots \ldots \ldots \ldots \ldots \ldots \ldots \ldots \ldots$

7.2 MAINTAIN $\ldots \ldots \ldots \ldots \ldots \ldots \ldots \ldots \ldots \ldots \ldots \ldots \ldots \ldots \ldots \ldots \ldots \ldots \ldots \ldots \ldots \ldots \ldots$

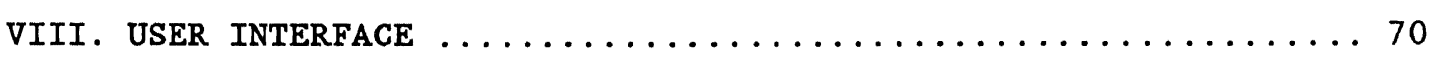

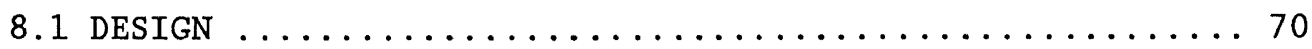

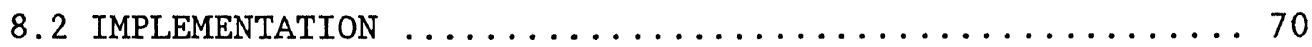

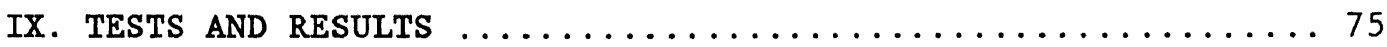

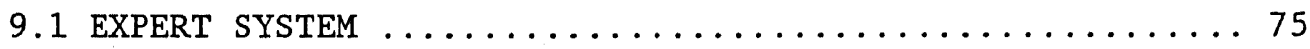

9.2 ON-LINE DATA ACQUISITION SYSTEM $\ldots \ldots \ldots \ldots \ldots \ldots \ldots 78$

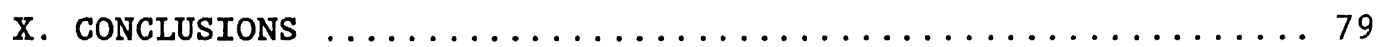

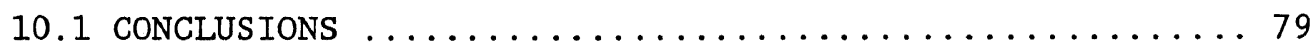

10.2 FURTHER DEVELOPMENTS $\ldots \ldots \ldots \ldots \ldots \ldots \ldots \ldots \ldots \ldots \ldots \ldots$

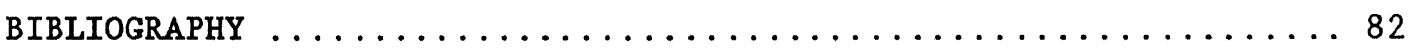

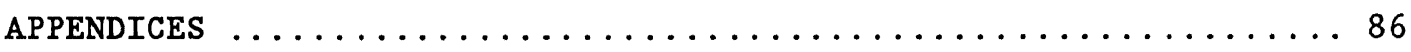

APPENDIX A.1 - SYSTEM COMPONENTS ............... 86

APPENDIX A.2 - ANAEROBIC TEST PROTOCOL .............. 87

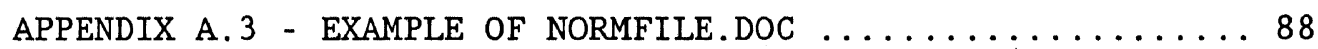

APPENDIX A.4 - PROGRAMMING THE A/D CONVERTER ......... 89

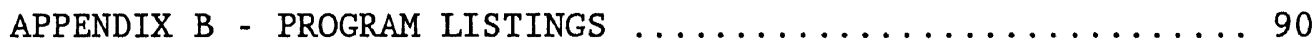

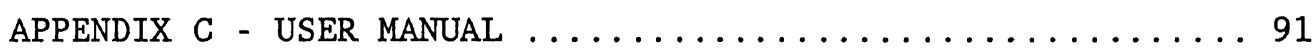

APPENDIX D - SAMPLE RECOMMENDATIONS .............. 97 
Figure 2.1 - Schematic of the application of EXFIT ......... 5 Figure 4.1 - Equipment for the on-line data acquisition system .. 14 Figure 4.2 - Equipment for the Wingate anaerobic test . . . . . . 17 Figure 4.3 - Timing diagram for detector on cycle ergometer .... 18 Figure 4.4 - Subject on treadmill undertaking Aerobic test ..... 21 Figure 5.1 - The overall structure of the software of EXFIT .... 26 Figure 5.2 - Schematic of profiling system ............ 30 Figure 5.3 - Sample output of the profiling system ......... 33 Figure 5.4 - Sample output of the performance system ......... 35 Figure 5.5 - Sample printout during the aerobic test ........ 39 Figure 5.6 - Sample graph produced during the aerobic test .... 40 Figure 5.7 - Sample printout of the report of the aerobic test . 45 results

Figure 6.1 - The overall structure of the software of EXFIT .... 49

Figure 6.2 - Internal structure of the knowledge in the advisory 54 system

Figure 6.3 - Sample output of the expert system for the ....... 59 metabolic status of the muscle (aerobic capacity) analysis

Figure 7.1 - Sample of the first screen in MAINTAIN .........66 66

Figure 7.2 - Sample of the second screen in MAINTAIN ........66 68

Figure 8.1 - Example of the user interface's flow from screen ... 72 to screen

Table 1 - Summary of responses from the coaches .........76

Table 2 - Summary of comments from the coaches .......... 77 


\section{ACRNOWLEDGEMENTS}

Thanks are due to: Associate Professor Graham Allen for the initial idea, suggestions during the project and the provision of the physiological expertise and knowledge required for the expert system, the Centre for the Assessment of Human Performance at the USQ for the equipment and purchase of additional equipment required to develop and complete the project, John Smith, Michael Murphy and George Blanas for assistance and supervision with the project, Ron Matthews and Keith Fleming for excellent craftmanship in the construction of any mechanical and electronic devices required, and finally Dina Parisi for considerable encouragement, support and motivation over the entire length of the project.

\section{DE C I A R A T O N}

This thesis has not been submitted in any form for another degree or diploma at any other University and the main text is an original work. 


\section{CHA P TE R I \\ I NTROD UCTION}

\subsection{OVERVIEW}

There is a need for a high level of sophistication in modern coaching and training techniques designed to enhance athletic performance. The effects of particular training schedules on athletes must be monitored closely and frequently. Regular and frequent physiological assessment of the athlete may be used to do this. For this thesis, fitness analysis refers to this assessment. However one of the problems in physiological testing is lack of feedback to the coaches and athletes of the results of the physiological tests and their implications for training and performance. The purpose of this project was to develop an expert system, EXFIT to bridge the gap between the scientist and the coach and facilitate the provision of scientific information in a systematic and coherent report to the coach.

\subsection{OBJECTIVES}

The primary objective of this project was to design a generalized expert system which would use the physiological parameters from a physiological analysis of the athlete to provide recommendations to improve the performance of the athlete. The athlete's coach may then use these recommendations to develop a specific training schedule suited to the particular athlete, and to the events for which the training is undertaken. The expert system has been designed to improve the interfaces between the physiologist and the coach and athlete. EXFIT does this by providing the athletes and coaches with advice in an economical and coherent fashion. Additionally, the automatic production of the report from EXFIT provides consistency in the style of communication, providing scientific information systematically and logically to the coach and athlete.

The system is designed to help the athletes understand the global aims that they should be achieving through training and the reasons why these aims are important. The expert system provides recommendations for a series of analyses, namely peak anaerobic 
power, total anaerobic power, aerobic power, onset of blood lactate accumulation, maximum blood lactate, blood analysis, muscle structure (fibre type), metabolic status of muscle - aerobic and anaerobic capacities.

A secondary objective was to develop an on-line micro-computer data acquisition system to provide some of the physiological variables required by the expert system. Provision was to be made for both manual and automatic acquisition of the relevant data from the tests from the peak anaerobic power, total anaerobic power and the aerobic power analyses. This data acquisition system has been developed as part of the project. The system acquires the data from the tests, performs the necessary calculations, displays the results, files the results onto a disc file for the athlete, produces a hard copy report for the athlete, plots the athletes change in performance between tests and profiles the athlete's results by comparing to the averages of the results by a group of athletes in the same sport.

This system will increase the overall efficiency of running these analyses and consequently increase the number of athletes that may be tested. In addition, for the aerobic power analysis, the automatic data acquisition system is an exemplary system for this type of analysis because it allows the athlete to be monitored at any user specified interval down to a minimum of fifteen seconds, and the results from each time interval printed and displayed graphically in real time. Compared to manual data collection, the automatic data acquisition system allows much greater control and ensures that the necessary data values are recorded only at the point at which the athlete is exercising at maximum work load. This point is accurately determinable with the data acquisition system.

The results of the test equipment not presently interfaced to the computer are manually collected and entered to EXFIT. The complete system is in use at the Centre for the Assessment of Human Performance at the University of Southern Queensland (USQ) in Toowoomba. 


\subsection{OUTLINE}

Briefly this thesis will be developed along the following lines:

Chapter 2 provides a general description of the system.

Chapter 3 summarizes the results of a literature survey of expert systems in the area of health and fitness.

Chapter 4 discusses the tests performed on the athlete during the fitness analysis. It also describes some of the associated hardware that is used in carrying out these tests.

Chapter 5 contains a discussion of the software developed for the online data acquisition system, calculations, display and for the reporting of results and the profiling of the athlete's performance.

Chapter 6 describes the expert system and associated software. The knowledge base has been split into data, information and knowledge as suggested by Debenham (1989a).

Chapter 7 outlines the software developed to allow the maintenance and updating of the expert system by the domain expert without the requirement for a knowledge engineer or programmer.

Chapter 8 describes how some of the basic principles of user interface design have been implemented in EXFIT.

Chapter 9 provides a description of any testing and verification of both the expert system and the data acquisition system of the fitness analysis software. 


\section{G E NERAL DESCRIPTION}

EXFIT is an expert system applied to the area of fitness analysis. A schematic of the application of the system is provided in Figure 2.1. EXFIT uses the results of a series of physiological assessments on the athlete to provide a report of generalized recommendations. This report is designed to improve the feedback from the results of the physiological assessments of the athletes to the coaches. The coaches may use the recommendations of the expert system in developing a specific training schedule suited for the particular athlete.

The expert system and computer are not intended to replace the physiologist nor the coach but instead to perform as an aid in improving the interface between the results of scientific assessment and their application in the field situation. The expert system has no role without the physiologist to apply the tests and interpret the results, and the coach who provides a practical interpretation and application of the results in the interests of the athlete.

The recommendations of EXFIT consist of one or more global aims for the athlete to achieve in order to improve performance. Generalized activities associated with these aims are recommended. If appropriate, an explanation of the effects of these activities is also provided along with any associated warnings and follow up procedures. The explanations outline any improvements and changes in physiology that should be noted to occur. The warnings detail any particular point that must be observed in order to prevent injury or any other detrimental effect. Follow up procedures specify how long before the athlete should return to be retested to check for any improvements.

The system has been designed and developed for an IBM-PC/XT/AT or compatible microcomputer. EXFIT is designed to provide an efficient and flexible user environment in which the user is guided through the entire session and any associated tests by a series of simple screenforms and window and menu interfaces. 


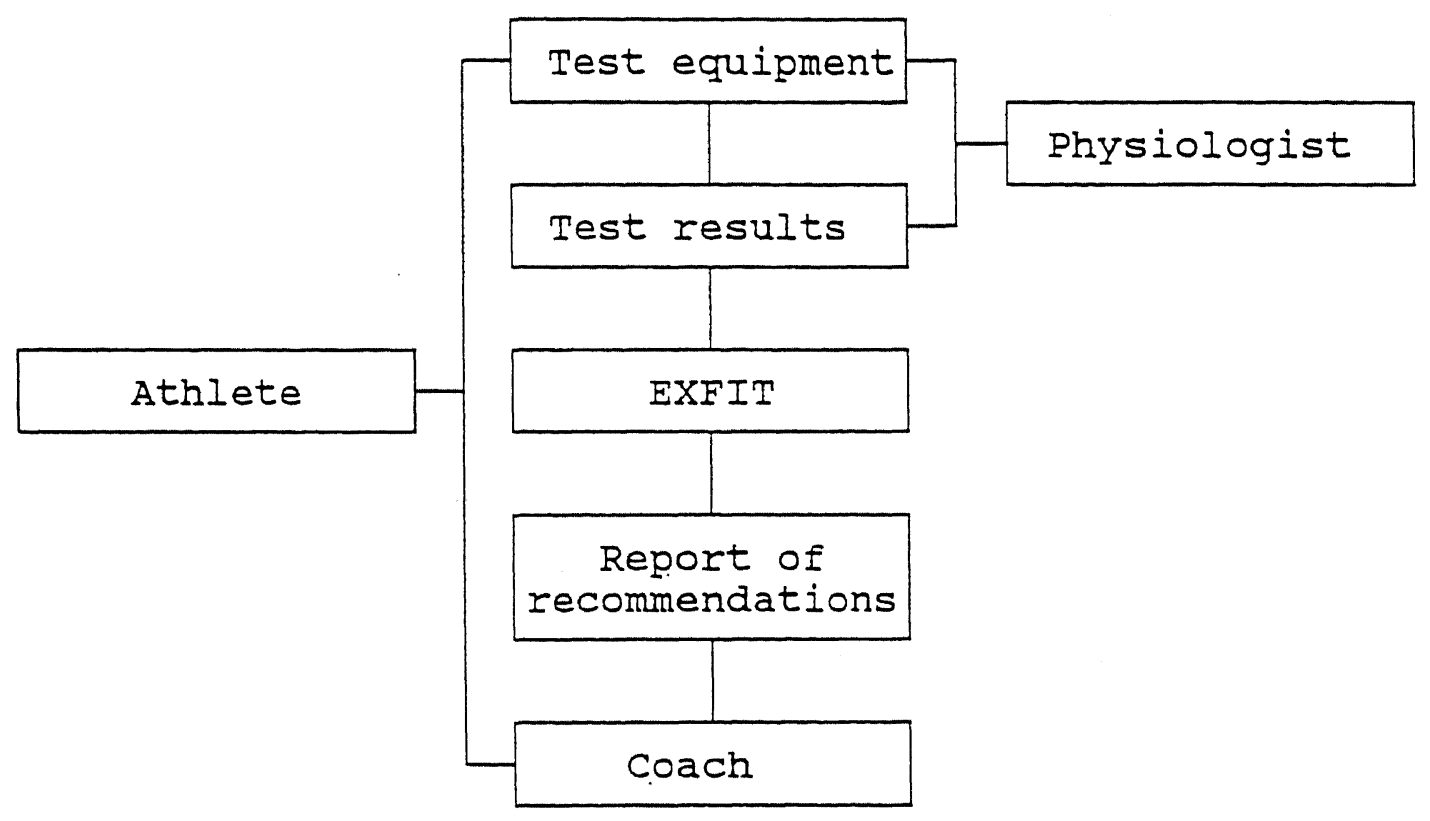

Figure 2.1 - Schematic of the application of EXFIT 


\section{CHA P T E R I I I}

\section{S URVEY OF RELEVANT RESEARCH}

Expert systems have been built to tackle many kinds of problems and they are successful in problem areas where clear algorithimic solutions do not exist (Parsaye and Chignell, 1988). They have been applied to many problem areas. One of the categories of the problems that may be addressed by expert systems are problems that require interpretation of data from sensors that interrogate particular situations (Waterman, 1986). In this category, the most likely area that EXFIT falls into is the area of health and medical interpretation systems that use the data from subject monitoring equipment to provide diagnoses followed by recommendations for treatment.

Townsend (1987) and Hawke (1988) list the reasons for expert systems' increasing popularity as:

- The increase in hardware capabilities with the decrease in hardware costs associated with more effective systems being designed has meant that expert systems are becoming available to run on personal computers .

- Expert systems provide an unemotional complete response at all times.

- Human experts are scarce with their services expensive.

- Human experts are busy and very much in demand.

- Expert systems can act as intelligent tutors for the instruction of students.

- Human experts are mortal.

Although in some cases expert systems can help a non expert make limited decisions, expert systems are also tools that can help a human expert make better decisions and to make these decisions more efficiently and consistently. This latter alternative is the case with EXFIT.

Perhaps the best known expert system in the health and medical interpretation area is MYCIN which assists physicians in diagnosing and treating meningitis and bacteremia infections. The system 
diagnoses the cause of the infection using knowledge relating infecting organisms with patient history, symptoms and laboratory test results (Shortliffe, 1976). Modified versions of MYCIN have been implemented for other domains including PUFF which diagnoses the presence and severity of lung diseases by interpreting the results of respiratory tests (Aitkens et al., 1983) and ONCOCIN which assists physicians in the treatment of cancer patients (Shortliffe et al., 1981).

Other examples in health and medical interpretation are:

- Garvan ES1 which provides clinical interpretation of thyroid assays in a pathology laboratory (Horn et al., 1985)

- AI/COAG assists in diagnosing blood diseases by analyzing and interpreting laboratory blood coagulation tests (Gaston et al., 1983)

- VM which monitors post surgical intensive care patients (Fagan et a1., 1984)

- BABY monitors patients in a newborn intensive care unit (Rodewald, 1984)

- INTERNIST acts as a diagnostic consultant for general internal medicine (Miller et al., 1982).

This list is not intended to represent a complete survey but rather * is an indication of the variety of expert systems that have been developed in the area of health and medical interpretation.

Although there is a wide range of expert systems in the area of health, expert systems applied specifically to fitness analysis and physical performance appear to be rare. This is the domain that EXFIT addresses. Vickers and Kingston (1987) describe an expert system being developed in ice hockey that consists of a laser videodisc controlled by an expert shell. The laser videodisc is used to portray the skills and tactics and other information used by a coach. Sainsbury et al. (1988) describe an on-line microcomputer system run on an Apple lle computer for the monitoring of physiological variables during rest and exercise. Owens (1985) has developed a system based on a Commodore 64 microcomputer system for monitoring heartrate, running a cardiovascular test, a muscular test 
and determining body fat. EXFIT has been extended beyond these systems to have an expert system provide recommendations for the athlete to improve performance. These recommendations are provided systematically and coherently in a report to the coach. 


\section{CHA P T R I V \\ DATA ACQUISITION}

\subsection{INTRODUCTION}

Before discussing the hardware and software used in this project, it is appropriate to describe the types of analyses and associated tests in EXFIT that may be carried out on the athlete. The analyses used were chosen after discussions with the Head of the Centre for the Assessment of Human Performance and after a visit to the Human Movement Studies Group at the University of Queensland in conjunction with reference to texts on physiological assessment such as Draper and Telford (1989), MacDougall et al. (1982) and deVries (1986). More information on why the analyses are important in the physiological analysis of an athlete is provided after the list of analyses below. Presently, EXFIT uses the following analyses to provide recommendations:

- Peak power analysis which utilizes the result of peak five second anaerobic power from the Wingate anaerobic test on the cycle ergometer and the level of body fat.

- Total power analysis is concerned with the level of total anaerobic power and the level of body fat.

- $\mathrm{VO}_{2}$ max analysis measures both the maximum level of oxygen consumed per minute per kilogram of body weight at maximum workload $\left(\mathrm{VO}_{2}\right)$ during the aerobic power test, and the level of body fat.

- Onset of blood lactate accumulation (OBLA) analysis considers if OBLA has been delayed with training or if there has been no change.

- Maximum blood lactate analysis uses the maximum lactate level in the blood.

- Blood analysis uses the results of tests on the levels of red blood count, ferritin, iron and hemoglobin.

- Fibre analysis looks at the results of tests on the muscle fibre of the vastus lateralis muscle. The size of FT, ST and the ratio of FTa to FTb are used in providing recommendations. 
- Metabolic status of muscle - Aerobic capacity looks at the levels of the aerobic markers SDH, CS, CYT-OX and the level of glycogen in the muscles.

- Metabolic status of muscle - Anaerobic capacity uses the levels of the anaerobic markers PFK, PHOS and the level of glycogen in the muscles.

Each of these analyses has a series of tests associated with it. The first three analyses can use either computer on-line acquisition or manual input of the data from the tests. The hardware and associated software written to do this are outlined in section 4.2 and in Chapter $V$. The remaining analyses are based on laboratory blood tests and muscle biopsies with the results entered manually into the expert system.

\section{on Line Analyses}

The peak and total anaerobic power analyses determine the anaerobic capacity of athletes. Lamb (1984) defines anaerobic capacity as "the ability to persist at the maintenance or repetition of strenuous muscular contractions that rely upon anaerobic mechanisms of energy supply". The anaerobic mechanisms of energy supply referred to by Lamb are a series of chemical reactions that take place within the associated muscles and do not require the presence of oxygen. Although these reactions produce the energy for muscles during intense exercise, the energy production and hence power output cannot be sustained. Lamb (1984) states that when "exercise is continued for more than about forty to sixty seconds, oxygen must be supplied by the blood to the working muscles", and aerobic (oxygen dependent) metabolism dominates energy production.

Anaerobic capacity is determined with the Wingate Anaerobic cycling test developed by scientists at the Wingate Institute in Israel (MacDougall et al., 1982). It is a thirty-second all-out cycling test to determine firstly the alactic or maximal anaerobic capacity over a five second interval and secondly the lactacid anaerobic capacity or the total energy output over the thirty seconds and the relative power decline which is an index of the fatigue rate. The 
test can be performed with legs or arms and the protocol for the test is outlined in Appendix A.2.

There are several limiting factors for most types of exercise which lasts longer than sixty to ninety seconds, namely: the capacity of the heart, lungs and circulation to deliver oxygen to the working muscles, and the chemical ability of tissues to use oxygen in breaking down fuels (MacDougall et al., 1982). Therefore any system providing recommendations to a coach requires an indication of the athlete's aerobic capacity. The most useful test of the functional capacity of the aerobic mechanism is one which measures the maximum amount of oxygen which can be consumed per unit of time by an athlete. The aerobic capacity is normally expressed as "the volume $(V)$ per minute $(V)$ of oxygen $\left(\mathrm{O}_{2}\right)$ which can be consumed by the organism at the maximum ( $\max$ ) workload which can be sustained for a criterion period of time and represented as $\mathrm{VO}_{2} \max ^{\prime \prime}$ (MacDougall et al., 1982).

Nagle (1973) maintains that treadmill running produces the highest values of $\mathrm{VO}_{2}$ max and is subject to the least differences between skill and efficiency of the subjects. $\mathrm{VO}_{2} \max$ is taken as the point at which the $\mathrm{VO}_{2}$ obtained peaks and levels. At this point a maximum heart rate should have been achieved by the athlete. Also the ratio of the volume of carbon dioxide produced per minute $\left(\mathrm{VCO}_{2}\right)$ to the volume of oxygen consumed per minute $\left(\mathrm{VO}_{2}\right)$, known as the respiratory exchange ratio (RER) should exceed one (Draper and Telford, 1989). These reference criteria indicate that the athlete is at maximum aerobic metabolism.

\section{Offline Laboratory Tests}

Lactic acid accumulates in the blood of an exercising athlete (McDougall et al., 1982). During exercise of increasing intensity, the power output at which levels of lactic acid rises significantly above normal resting levels is termed as the onset of blood lactate accumulation (OBLA). The OBLA is a very critical factor in determining the athlete's potential for sustaining prolonged physical exercise. The OBLA can be delayed with training (MacDougall et al., 
1982). Trained athletes can produce greater amounts of lactic acid because they have greater stores of muscle glycogen to break down to lactic acid and because they can better tolerate increased levels of lactic acid (Lamb, 1984). The maximum blood lactate analysis of EXFIT takes into consideration these levels in providing recommendations.

In addition to lactate accumulation in the blood there are other characteristics of the blood that are important for aerobic endurance exercise. Working muscles require a supply of oxygen, which is transported by the hemoglobin in the red blood cells. Iron is a necessary component of hemoglobin and plays an essential role in oxygen transport (Fox, 1984). From this it follows that the number of red blood cells and the amount of hemoglobin in those cells determine the amount of oxygen which can be transported to the working muscles. Blood characteristics are important in relation to the aerobic endurance performance of the athlete.

Human skeletal muscle has a mixture of two types of fibres which are distinguished according to their contraction profiles. Fast twitch (FT) fibres, (subdivided into FTa and FTb) attain peak tension rapidly (within $20 \mathrm{msecs}$ ) and are important in events requiring short powerful bursts of power. Slow twitch (ST) fibres complete a contraction slowly (60 - 80 msecs) and are better adapted for endurance events (Lamb, 1984). The classification of fibre types is based on an analysis of a sample of muscle tissue obtained by a needle biopsy procedure. As a result of specific training, the appropriate type of fibre will undergo hypertrophy and thereby increase the cross sectional area of the muscle fibre.

The metabolic status analyses used in EXFIT refer to the series of chemical reactions that take place within the body to break down glycogen to provide energy. Aerobic metabolism is the series of chemical reactions that require the presence of oxygen and anaerobic metabolism is the reactions that do not require the presence of oxygen (Fox, 1984). Several enzymes are used regularly as indices of either aerobic or anaerobic status of muscle. For anaerobic metabolism these enzymes are phosphofructokinase (PFK) and 
phosphorylase (PHOS) and for aerobic metabolism these are cytochrome oxidase (CYT-OX), succinate dehydrogenase $(\mathrm{SDH})$ and citrate synthase (CS) (Brooks, 1985).

\subsection{EQUIPMENT}

For the on line analyses the tests are run with the athlete using a computer controlled data acquisition system developed as part of this thesis. The details of the system are schematically outlined in Figure 4.1 and the components listed in Appendix A.1. This system provides the physiological data required for these analyses by the expert system. Data entered manually include the athlete's personal details, body weight and body fat, the barometric pressure, the ambient temperature and the collection time frame for the tests. The microcomputer used is an IBM compatible with a 5.25 inch floppy disc drive, a $42 \mathrm{M}$-byte hard disc and $512 \mathrm{~K}$-byte of random access memory (RAM), a commonly available machine. 


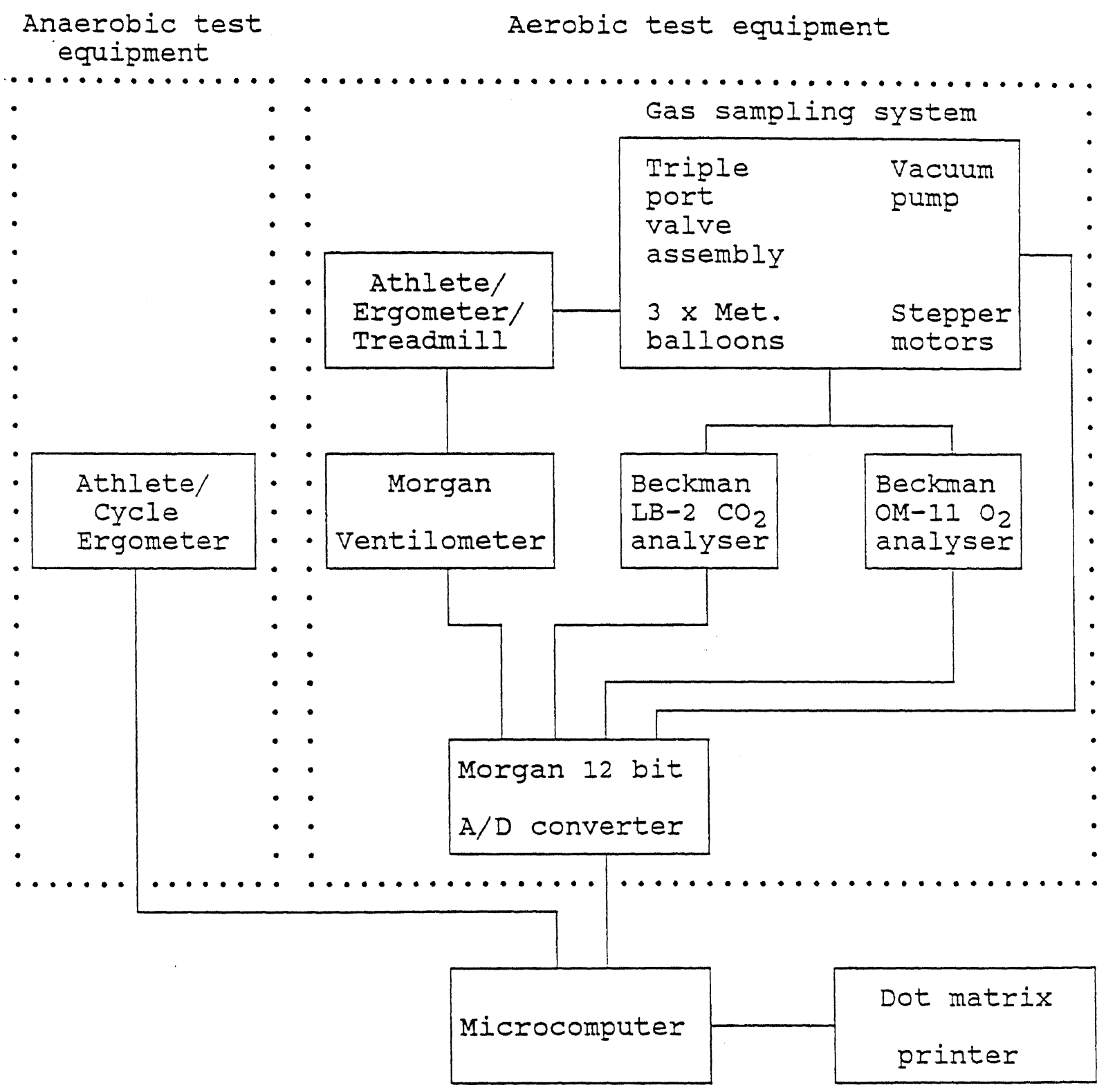

Figure 4.1 - Equipment for the on-line data acquisition system 


\subsubsection{ANAEROBIC TEST EQUIPMENT}

In Figure 4.1, the Wingate anaerobic test is based on the cycle ergometer consisting of a fixed wheel flywheel on which a specific tension or load can be set. A Gallium Arsenide infra-red emitting diode and sensor to detect contrasting spots on the cycle wheel were installed on the ergometer. The contrasting spots are black dots attached at regular intervals around the flywheel. The associated circuitry with the detector was designed and built by White (1989) according to the specifications provided by the author of this thesis. The circuitry provides a square wave that changes in polarity each time a pre-defined number of contrasting spots has been detected. This square wave is input to the computer via the ACR pin of the parallel I/O port. Any pin that allows a signal to be sent from the device to the computer could have been used. Although it would have been logical to use the games port instead of the parallel port, there is no games port installed on the computer in use thereby necessitating the use of the parallel port. The software that was written to collect the data is described in Chapter 5. The protocol for the test is outlined in Appendix A.2.

A photograph of the equipment for this test is shown in Figure 4.2. The box housing the detector and circuitry may be seen attached to the front fork of the ergometer. To run the test, the athlete pedals the ergometer as fast as possible to overcome the inertia of the flywheel and obtain maximum cadence. At the same time the computer program (developed by this author) is initialised to select the number of contrasting spots on the wheel that the circuitry must detect before the square wave output changes in polarity. The software measures the rate of detection of contrasting spots on the wheel and instructs the hardware to change the polarity of the square wave at the detection of a given number of spots, namely every spot, every second spot, every fourth spot, or every eight spot as shown in Figure 4.3. The software transmits a $0,1,2$ or 3 on the DO and D1 lines of the parallel port to select the appropriate 'gearing'. Once selected the gearing remains constant for the duration of the test. The selected gearing is displayed on the LCD display on the box housing the circuitry. This flexibility ensures that the square wave 
being input to the computer is never changing its polarity at a rate which is too fast for the computer software to detect. In this manner the equipment can be used to test subjects over the complete range of capabilities. A gearing that changes the polarity of the square wave at the detection of every fourth spot is more than adequate to cope with the expected absolute maximum of 200 revs per minute of the pedal crank. 


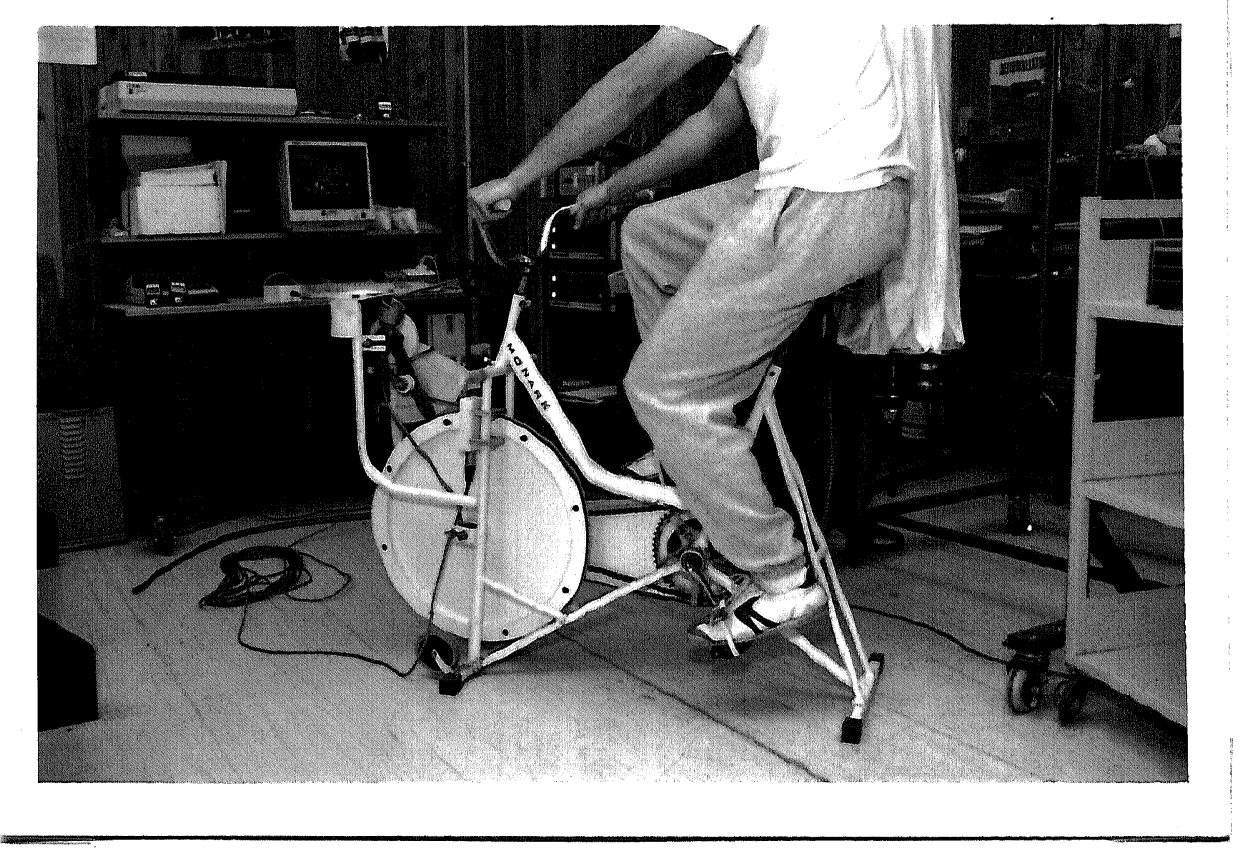

Figure 4.2 - Equipment for the Wingate anaerobic test 


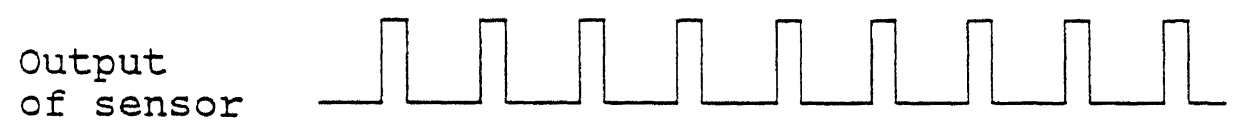

Input to computer
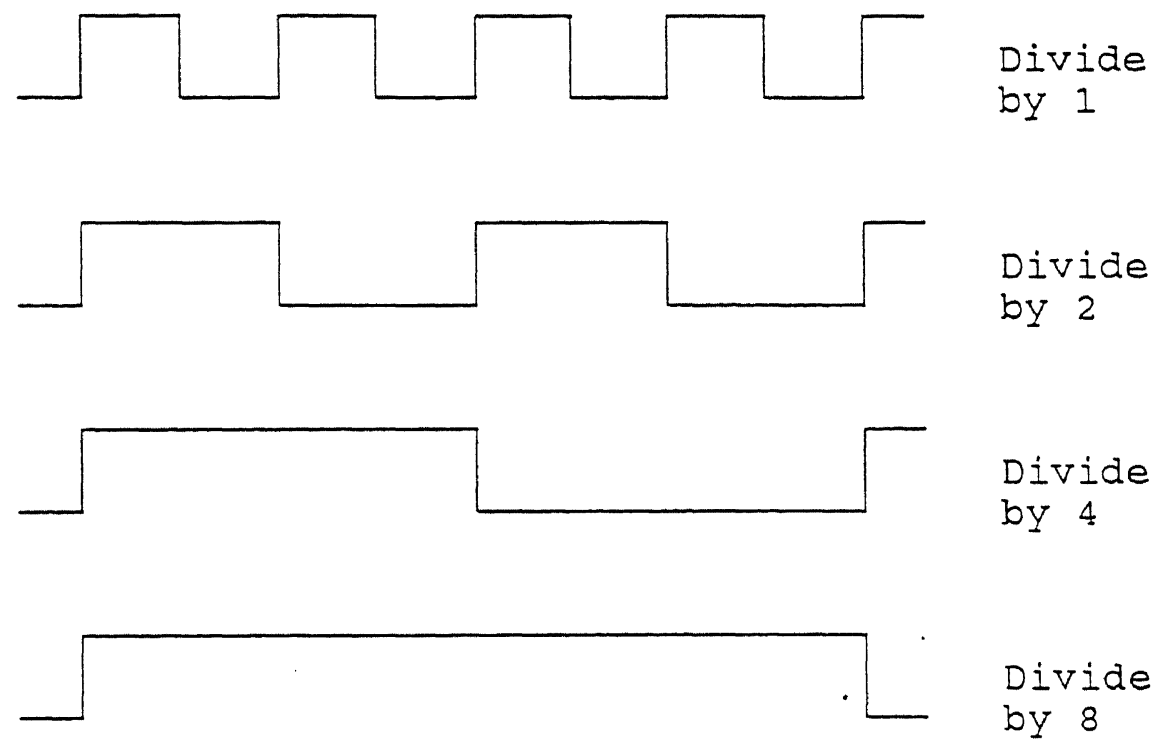

Figure 4.3 - Timing diagram for detector on cycle ergometer 
When the athlete attains maximum cadence, a predetermined load (calculated by the software from the athlete's body weight and the optimal load table in Appendix A.2) is applied to the flywheel. Once the final load has been applied and a key struck on the computer keyboard to signal the start of the test period, the computer software continuously monitors the parallel port for thirty seconds as the athlete continues to pedal at maximum output. The computer beeper sounds loudly to signal the termination of the thirty second test period. On completion of the test the software provides the total number of pedal revolutions over the test and the peak number and lowest number of pedal revolutions in any five second period during the test. The software uses this and the value of the load applied to calculate the total anaerobic power, peak five second and lowest five second anaerobic power exerted along with the power decline.

\subsubsection{AEROBIC TEST EQUIPMENT}

For the second part of the on-line data acquisition system, the software written by this author controls the measurement of the volume of air inspired by the athlete on the treadmill by the Morgan ventilometer (Morgan Inc., 1988b) and the recording of carbon dioxide $\left(\mathrm{CO}_{2}\right)$ and oxygen $\left(\mathrm{O}_{2}\right)$ concentrations by the gas analysers (Beckman Instruments Inc.). Figure 4.4 shows a subject on the treadmill with the gas analysers and ventilometer in the equipment rack. The rectangular frame holding the triple port valves and the meteorological balloons was designed and built for this project by the mechanical workshop at the USQ so that the assembly holding the valves and balloons can be set at different heights. This provides the flexibility necessary for testing athletes on the treadmill or the cycle ergometer.

The gas sampling system has been developed according to the description provided by Sainsbury et al., (1988), with several modifications made by the author of this thesis to improve the system. The changes outlined in Parisi and Allen (1991b) have been made to enhance the systems speed, utility and flexibility and include the following: 
- Starting the test at the end of an inspiration and waiting for the end of an inspiration at the end of a time frame before rotating the triple port valve, thereby ensuring that there is always a whole number of inspirations in a time frame. The user designates a time period and the computer responds to the end of the respiratory cycle closest to the end of the prescribed collection period.

- The system has been modified to execute on an IBM compatible PC instead of the Apple computer used by Sainsbury et al. (1988).

- Usage of a vacuum cleaner with the filters and bag removed rather than the vacuum pump. This is purely as a cost saving measure. The suction rate of the vacuum cleaner was tested and easily coped with the expected maximum flow rate of 240 litres/minute.

- The time frame interval may be altered during test execution without the necessity of restarting the program with a new collection interval, thereby enhancing the utility of the system.

- During test execution, a graph may be displayed of the results versus time frame number. A benefit of such a display is that it provides the user with immediate demonstration of the physiological stress on athletes with the facility of terminating the test at an appropriate time thereby minimising the magnitude of stress and discomfort imposed on the athlete.

- At test completion a report can be printed with a history of the athlete's past test results.

- Profiling the athlete's results from the latest test with the means of the results of other athletes in the data base.

- Plotting the athlete's change in performance in selected areas as a function of the test date.

The software to implement these changes is described in detail in Chapter 5 . 


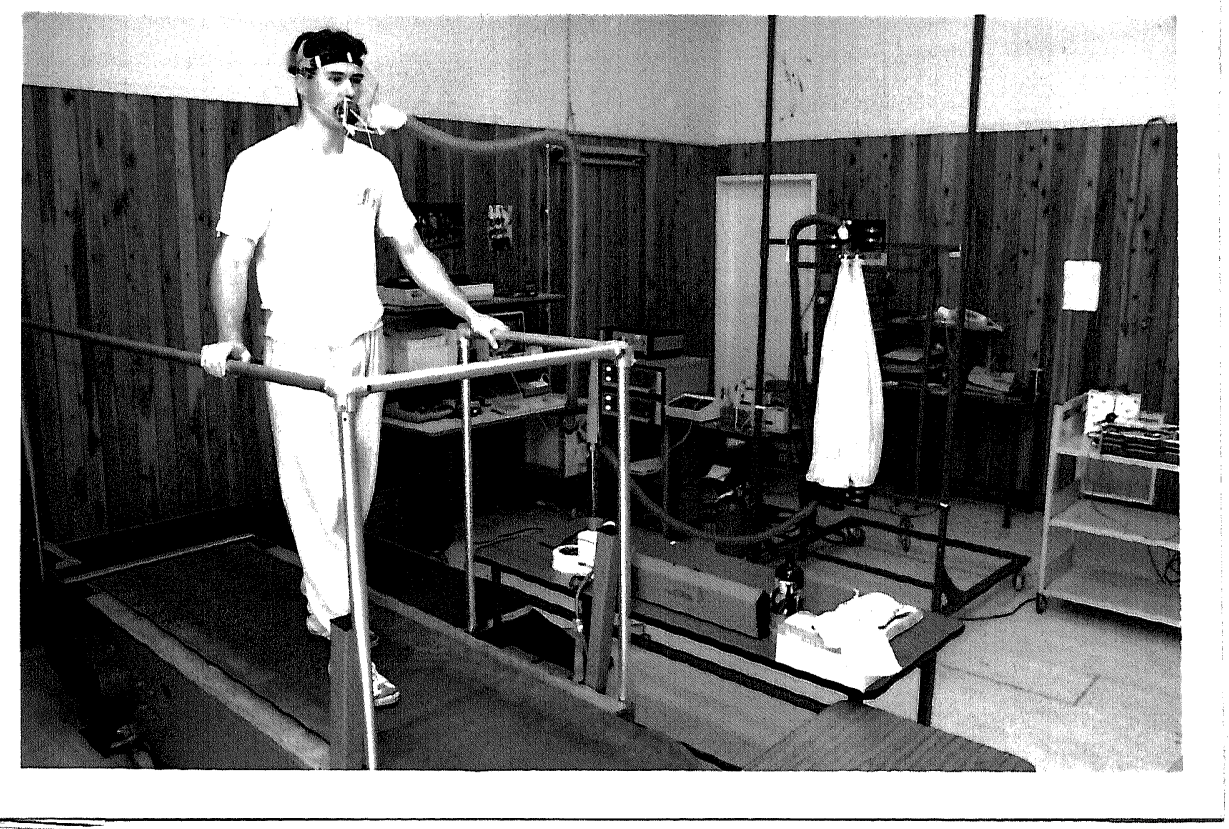

Figure 4.4 - Subject on treadmill undertaking Aerobic test 
A problem was encountered in that the balloon being evacuated by the pump was being sucked up the pipe attaching the pump to the balloon. The consequence of this was that the evacuation of the balloon was restricted. This problem was overcome by this author by fitting a $19 \mathrm{~mm}$ diameter PVC tube inside the balloons. This tube is attached to the suction pipe at the top of the balloon and runs the entire length of the balloon. The tube has a series of holes drilled down its entire length which serve to spread the point of suction and so overcome the problem of the balloons being sucked up the tube by the vacuum.

The gas sampling system works as follows: at any time, one balloon is being filled with expired air, one is being analysed and one is being evacuated by the pump. At or close to the end of the designated time frame and at the end of an inspiration close to the end of this time frame, the balloons are rotated by computer control via the triple port valve controller (Danz Instrument Service, 1989) and the process is repeated. The test continues until the $\mathrm{VO}_{2}$ for each time frame platforms at a maximum. Provided that a maximum heart rate is achieved and the RER is greater than one, the athlete is exercising at the maximum workload.

The analogue signals from the measuring instruments are input to a Morgan 12 bit analogue to digital (A/D) converter (Morgan Inc., 1988a) which allows for up to 16 analogue inputs and two bytes of digital output. The junction box has a series of test points which proved useful in the setup and debugging of the system. The digital output of the A/D converter is also via this junction box. A second junction box houses a relay to drive a 240 volt socket for the vacuum pump from the digital output of the $A / D$ converter. These junction boxes were designed and built by the electronics workshop at the USQ according to the specifications provided by this author. Twelve bit resolution was required to provide the necessary resolution for the readings from the gas analysers.

The analogue signals range from 0 to 5 volts and have a significant level of random noise superimposed on them. The effect of this noise is minimized by the software sampling the appropriate channel a large 
number of times, say 100 times and taking the mean. The ventilometer is sampled by the $A / D$ converter at the end of the inspiration closest to the end of the time frame. The gas analysers are sampled half a time frame later. Sampling half way through the next time frame allows time for the gas analysers' signals to stabilize. Presently only the following channels of the A/D converter are used:

$$
\begin{aligned}
& 1 \text { - percent oxygen } \\
& 4 \text { - percent carbon dioxide } \\
& 8 \text { - volume inspired } \\
& 9 \text { - number of inspirations }
\end{aligned}
$$

The capability exists for future expansion of the test equipment interfaced to the computer.

The digital outputs provided by the A/D converter are used to reset the ventilometer at the beginning of each test, to rotate the stepper motors 120 degrees and to control a relay to switch on and off the vacuum pump. In order to reset the ventilometer it requires a 5 volt level to be grounded. The stepper motors will rotate by one step if a 5 volt level is grounded for approximately three milliseconds (Danz Instrument Service, 1989). Each step corresponds to 0.6 degrees. The signal level is raised low and high as a square wave with a six milliseconds period, a total of two hundred times to rotate the motors 120 degrees. 


\section{CHA P TE R V}

\section{FIT NES S TESTING SYSTEM}

\subsection{STRUCTURE}

The overall structure of the software for the complete system is shown in Figure 5.1. The software consists of programs subordinate to the main program called EXFIT. EXFIT is the top level of the system and calls six other programs with no direct communication between the lower level programs. Each program is an executable file capable if required of being run independently of the other programs. This modular nature of the software has several advantages (Tanenbaum, 1984, Kruse, 1987), namely:

- Modifications and updates can be made to one program without requiring changes in the others.

- Each executable program file is loaded into memory as required and so reduces the memory requirements. The complete system is never loaded into memory.

The complete set of programs are:

- EXFIT, the main program and expert system also handles user interaction and controls the overall action of the system.

- MAINTAIN allows changes to be made to the data and information of the expert system.

- COMPARE performs two functions. The first is to compare the athletes present performance with the average performance of athletes in the same sport. The second is to plot the athlete's past and present performance as a function of the date of the test to enable a speedy assessment to be made of the athlete's change in performance with the recommended aims and activities from the expert system.

- AEROBIC obtains the results from the aerobic test equipment, performs any necessary calculations and files the results onto a disc file.

- ANAEROBIC is the same as the aerobic test except for the anaerobic part of the test.

- PRINTREP prints a report of the athlete's details and test results.

- DISPLAY displays the records from the athlete's file on the screen 
and if required allows the deletion of any records that are no longer required.

This Chapter deals with the software for the fitness testing system of EXFIT that is shown highlighted in Figure 5.1. The other components, namely the expert system, the maintenance program and the user interface are discussed in Chapters 6,7 and 8 respectively. 
EXFIT: The Advisory System

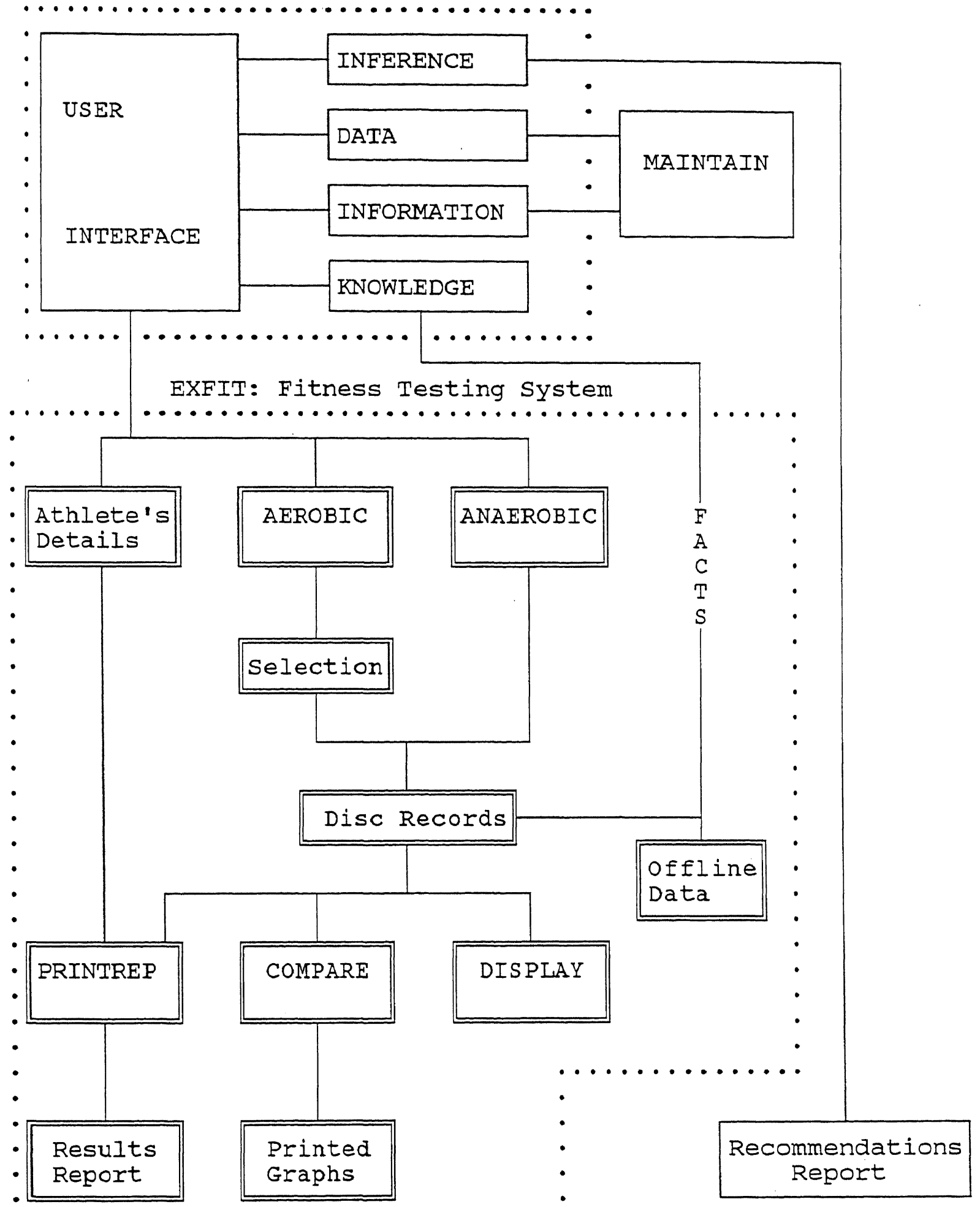

Figure 5.1 - The overall structure of the software of EXFIT 


\subsection{CHOICE OF SOFTWARE}

The main program EXFIT, written in Turbo Prolog exploits its non procedural nature and ability to backtrack. The user interface facilities in the Turbo Prolog Toolbox (which is a collection of over eighty predicates), (Borland, 1987b) are utilized in the user interface. With the exception of MAINTAIN, all of the subordinate programs are written in Turbo Pascal 4.

Turbo Pascal was chosen as the procedural language to use for the subordinate programs for the following reasons:

- It was a language with which the author was already familiar and a compiler to run on an IBM PC with associated documentation was readily available.

- Pascal is a language that encourages the programmer to write programs that are modular in design and can be easily understood by other programmers (Dale and Weems, 1987).

- Turbo Pascal provides efficient implementation of facilities such as graphics, file handling, calculations and access to facilities of the operating system (Borland International, 1987a, Banks, 1988).

\subsection{DESCRIPTION OF SOFTWARE}

The following sections deal with the components in the fitness testing system that are shown highlighted in Figure 5.1.

\subsubsection{DISC RECORDS}

The filename for the disc records for each athlete is constructed from the first initial, the surname and the extension FOR or DAT according to whether the file contains the athletes details (FOR) or records with the athletes test results (DAT). For example, the athlete Norm Occker would have his details stored on the file NOCCKER.FOR and the test results in NOCCKER.DAT. A more sophisticated method of athlete identification would be required at a larger testing facility, however the above method has proved adequate at the USQ.

The test results are stored in a binary file with each record 
containing a series of fields denoting all the results from a test. This file is stored in binary format to provide a minimal form of data encryption. The purpose of the file is to provide a record of the athlete's results from each test. This provides a mechanism for monitoring the athlete's progress. Each record has a flag to denote the type of test to which it relates. Specifically an ' $A$ ' denotes a record for the aerobic test and a ' $W$ ' denotes a record for the Wingate anaerobic test. In this fashion, if the fitness analysis system is extended, additional records may be included with appropriate flags. The records are stored in chronologically decreasing order with the most recent records stored first. As described further in the following sections, PRINTREP, COMPARE and DISPLAY all use the disc records in producing both a printed report of the athlete's results and printed graphs of the comparison of the athlete's results against other athletes and of the athlete's change in performance with each test.

\subsubsection{OFFLINE DATA}

The offline data is from the laboratory blood tests and muscle biopsies as outlined in Section 4.1. The results from these tests are manually entered into the advisory system via a screen menu for each test. Each screen menu provides the option of entering three possible values, namely higher, lower or comparable to the average of the results for that test by a group of athletes in the same sport. The appropriate level of the athlete's test result is entered via the menu.

\subsubsection{COMPARISON SYSTEM - COMPARE}

The source code listing for the comparison system called COMPARE is given in Appendix B. This program is designed to perform two functions. The first is to profile the athlete's results from the latest test with the means of the results of the other athletes in the database. This is a ready indicator of the areas in which the athlete needs improvement. The second function is to plot the athlete's change in performance in a selected area as a function of the date of the test. This plot is a simple indicator of whether the training recommended by the expert system and carried out by the 
athlete is having any effect. Both of these plots have been provided following a request by the physiologist.

\subsubsection{PROFILING SYSTEM}

This section outlines the first function of the comparison system which profiles the athlete against other athletes in the same sport. A schematic of the profiling system is given in Figure 5.2. Before any profiling is done, the user has the option of the program recalculating the means for the group of athletes. The calculation of the means does not have to be redone before each comparison. They can be calculated on a periodic basis whenever there has been a major update to the data stored in the files on disc for each athlete. 


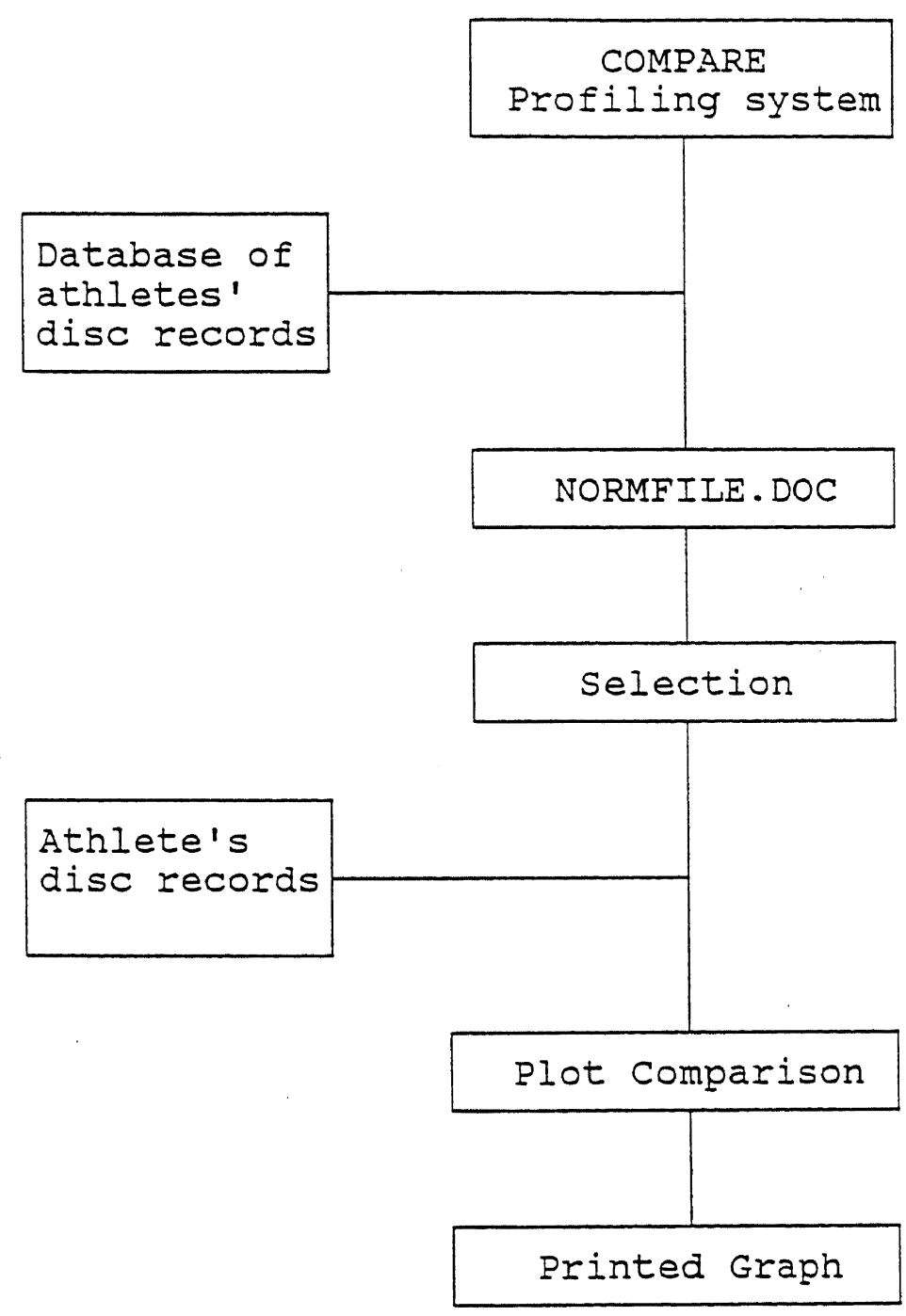

Figure 5.2 - Schematic of profiling system 
If the user opts to recalculate the means, the program will scan the database of disc records for all athletes and prompt the user as to which files are to be used. The program utilizes the Turbo Pascal built in procedure FindFirst to search the specified directory for the first entry matching the specified file name of $*$.FOR. The built in procedure FindNext is then used to return the next entry that matches the name and attributes specified in the previous call to FindFirst.

For each file found, the filename and athlete's age are displayed and the user is asked whether the data in the file are to be used in calculating the means. The athlete's age is displayed as the user may be calculating the means on an age selection basis. As an example, the user may want to calculate the means for only cyclists younger than thirty years. For this reason the age is displayed and the user is allowed the choice of using the file. The data discs of test results are sport specific. The files for athletes who are, for example, cyclists are on one disc. The files for athletes in another sport are on a second disc and so on. Once a particular disc for a sport is full, a second disc is started. For this reason, once all the files on a disc have been scanned, the user is asked if it is necessary to repeat for a second disc. The reason for grouping the files in the above fashion is to build up databases of results according to particular sports.

As outlined in Figure 5.2, the calculated means are stored on a file NORMFILE.DOC. An example of this file and the values contained in this file is provided in Appendix A. 3 .

Before plotting the comparison, the user selects the variables to be compared. The possible list of variables from the tests are displayed in a menu and the user can select up to a maximum of twenty one variables at a time for the comparison. The maximum is imposed as this is all that will fit vertically on the screen after allowing for titles and labels on the axes. The program scans the athlete's disc record and NORMFILE.DOC, selecting from these the values for the variables chosen. A plot of the comparison is now displayed on the 
screen. An example of this comparison is shown in Figure 5.3. The means for the group of athletes are represented as $0 \%$ with the athletes results plotted relative to this up to $20 \%$ either side. If the difference is greater than $20 \%$ a ' ' $*$ ' is plotted at the appropriate extremity. The variable plotted on each line and the athlete's results for each one are displayed on the left hand side of the graph. 
ATHLETE PROFILING SYSTEM

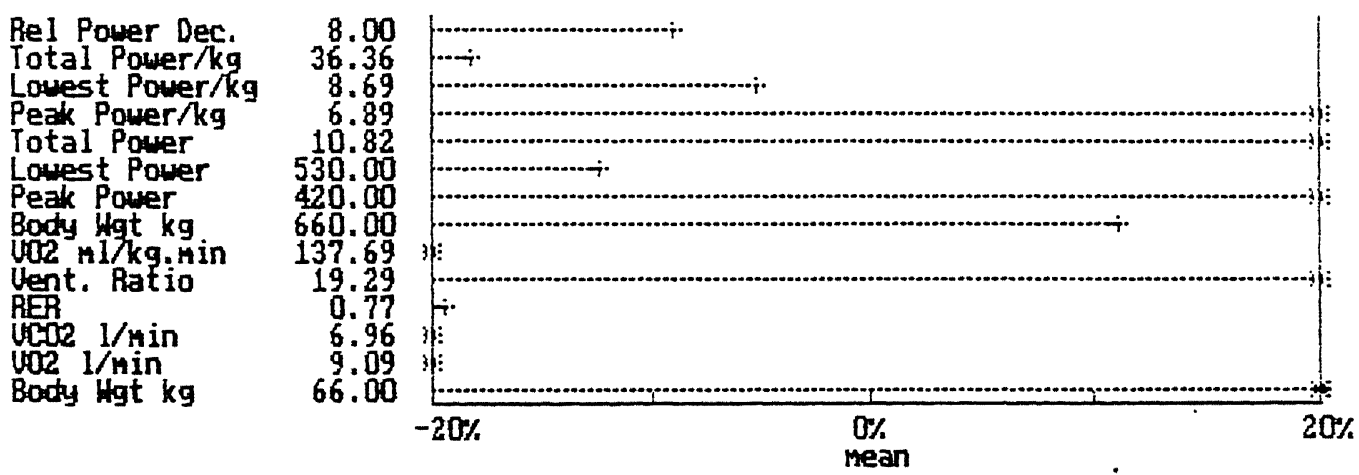

Figure 5.3 - Sample output of the profiling system 
Once the comparison is plotted on the screen, the user has the option of pressing <F10> to obtain a hard copy on the dot matrix printer or of returning to the main menu. The procedure for printing high resolution graphics on dot matrix printers was written according to the description provided by Bridger and Goresky (1985).

\subsubsection{PERFORMANCE SYSTEM}

The user selects the file to be used in a similiar fashion to the previous section. The variable to be plotted is selected from a menu. The software reads the selected file and plots the test results of the variable as a function of the date of the test being performed. All the test results for that variable up to a maximum of nine are plotted and may be printed. If there are more than nine records of results, only the latest nine are plotted as any more than this causes the annotation on the $\mathrm{x}$-axis to be wider than an $\mathrm{A} 4$ page. An example graph is shown in Figure 5.4. 
ATHLETE'S PERFORTANCES

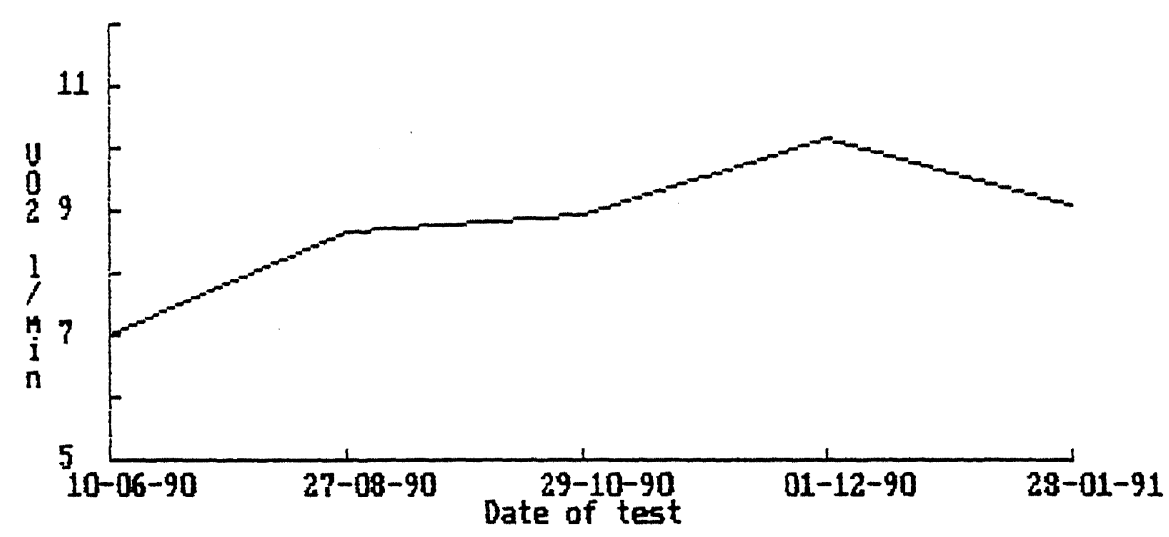

Figure 5.4 - Sample output of the performance system 


\subsubsection{AEROBIC TEST - AEROBIC}

The aerobic test module in Figure 5.1 forms a part of the automatic data acquisition system. The equipment for this test is described in Section 4.2.2. At the start of this test, the software displays a screen form for the athlete's details. It is necessary to enter the athlete's surname and first initial as these are used in producing the filename for the results. The date and time of the test are entered automatically from the system. Part or all of the other information on the form may be omitted if not required. The source code for the software for this test, called AEROBIC is provided in Appendix B.

Automatic or manual data entry mode are selected from a menu. If the manual data entry mode is selected, the user enters the data via a screen form. For the automatic data entry, the ambient temperature, barometric pressure and athlete's body weight are presently not possible to enter automatically and are entered manually into a screen form. As outlined in section 4.2.2, the data from the test equipment are input via an $A / D$ converter. The program requests whether calibration of the $A / D$ converter is required; if required, the user is prompted to enter the values displayed on each of the instruments so that a calibration factor may be determined. It is not necessary to calibrate the A/D converter each time the software is executed as the calibration factors are stored on a disc file for future use.

The A/D card uses eight consecutive I/O address spaces starting at the base address $0710 \mathrm{H}$ or 1808 (Morgan, 1988a). The usage and programming of the eight addresses is detailed in Appendix A.4. A channel is sampled by sending the channel number ( 0 to 15 ) to the $A / D$ channel select and the value converted is returned via two bytes. Bit 7 of the most significant byte contains the status which is checked by the software to indicate if the conversion has been successful. Digital I/O are simple read and writes to the appropriate addresses. For this project, output through port $B$ is all that is used.

The software instructs the user to strike any key to start the test. 
Once started, the software waits for the end of an inspiration before commencing a time frame. The end of an inspiration is determined by monitoring the respiration number on the $A / D$ converter from the ventilometer. This number increments only at the completion of an inspiration. Following the start of a time frame, the software monitors the number of clock ticks on the internal clock (Norton, 1986) to determine the end of each time frame. At the completion of the inspiration closest to the end of a time frame, the software obtains the value on the A/D converter channel for the volume. This ensures that there is always a whole number of inspirations in a sample interval.

The volume on the ventilometer increments with each inspiration. The volume inspired over the time frame is calculated by subtracting the value on the ventilometer at the end of the previous time frame. The digital display of volume on the ventilometer increments up to a total volume of 999.9 litres but the analogue output on the computer socket increments only to 99.9 litres and then overflows to zero (Morgan, 1988b). Similiarly the digital display of the number of respirations increments to 999 but the analogue output has a maximum of 99 overflowing to zero. Both these overflows are accounted for in the software.

At the end of the time frame, the triple port valve is rotated by sending the appropriate byte to the triple port valve controller via the port $B$ output of the $A / D$ converter. As outlined in Section 4.2.2, the analysers are sampled halfway through the next time frame, the calculations performed and the results displayed. A sample of the values computed for each time frame are displayed in Figure 5.5. Note that the time for each record varies due to each time frame starting and ending at the end of an inspiration. The values in the Figure are computed according to Fox and Mathews (1981). These values are printed on a dot matrix printer if one is connected and on line. To prevent the system hanging up if a printer is not connected and on line, the software first checks for this. In addition the user specifies whether these values are to be displayed on the screen or whether a graph is plotted of $\mathrm{VO}_{2}$ and respiratory exchange ratio 
(RER) as a function of the time frame number. A sample graph is depicted in Figure 5.6.

The solid line on the graph in Figure 5.6 is a plot of the $\mathrm{VO}_{2}$ and the dashed line is a plot of the RER. These two variables were selected because as outlined in section 4.1, two of the requirements for determining $\mathrm{VO}_{2} \max$ are that the $\mathrm{VO}_{2}$ levels out to a maximum and the RER exceeds one. This illustrates the advantage of an online system that calculates $\mathrm{VO}_{2}$ and $\mathrm{RER}$ at the end of each time frame during a test. The point at which the athlete is exercising at maximum workload before the athlete becomes fatigued and $\mathrm{VO}_{2}$ starts to drop can be determined accurately. This means that a more accurate assessment of the aerobic capacity can be made. 


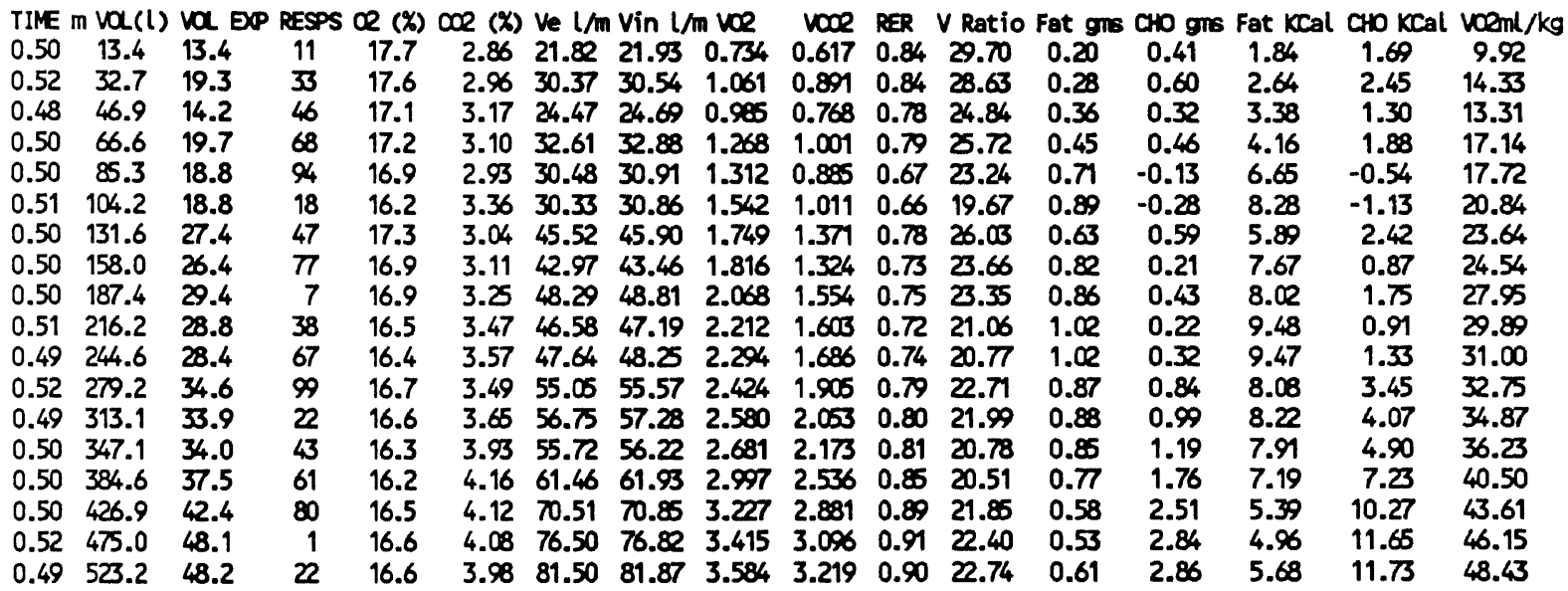

Figure 5.5 - Sample printout during the aerobic test 
U02 and RER us Tine

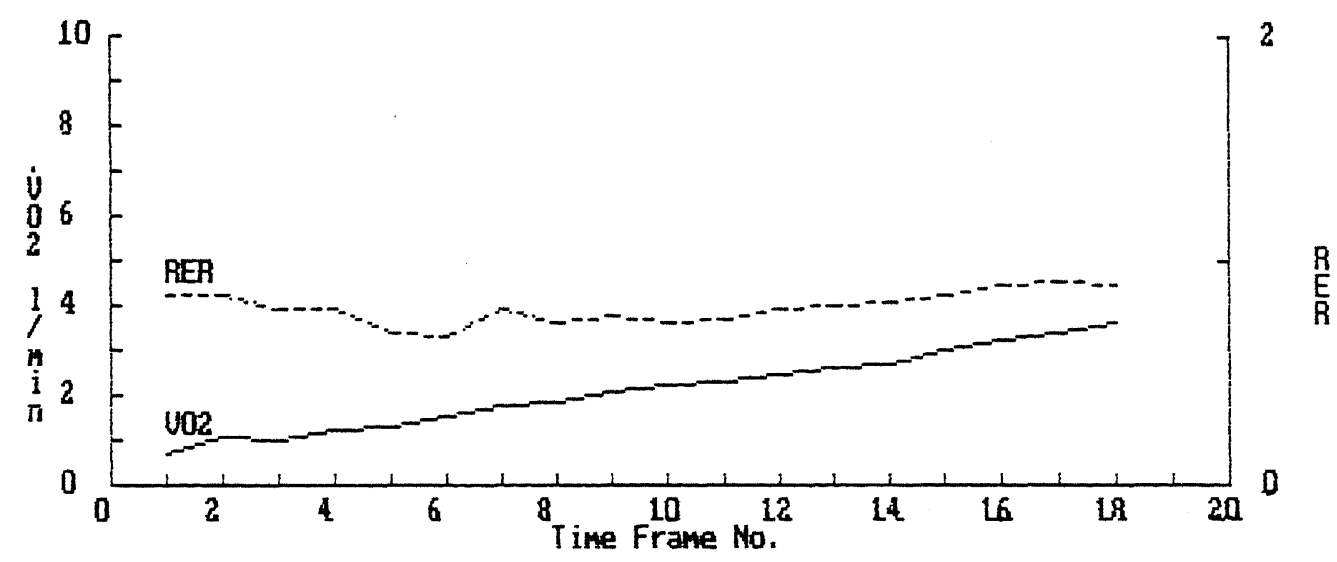

Test running - press ESC to end

Figure 5.6 - Sample graph produced during the aerobic test 
The values for up to a maximum of 20 time frames are stored in memory as the test runs. The value of 20 was chosen because a test of maximum aerobic capacity rarely exceeds 20 time frames. A test exceeding 20 time frames has been provided for by using a cyclic buffer whereby the latest test results overwrite the oldest results in memory. The test continues until the user presses the <ESC> key to end the test. The software waits till the end of the time frame to conclude the test.

For some applications it may be necessary to change the time frame interval. This occurs for a test that monitors the athlete during periods of rest, prolonged exercise or a combination of rest and exercise. During the periods of rest, the sampling time frame interval is much longer, for instance five minutes compared to thirty seconds during periods of exercise. The longer time frame interval during rest periods allows the collection of a sufficient volume of expired air. Pressing the $\langle$ F10 $\rangle$ key allows the user to change the time frame interval during the test.

The values calculated for each time frame are displayed on the screen and from a menu the user selects the record or records at which the athlete was exercising at maximum oxygen uptake. If more than one record is selected, as may be the case if the athlete is exercising at maximum oxygen uptake over more than one time frame, the averages for each variable in the records are calculated. As depicted in Figure 5.1, the record selected or the calculated averages are written to the athlete's file on disc. The records are stored in chronologically decreasing order. It is important to store the athlete's results as in order to obtain the best possible performance from athletes, the training stress must be optimized on an individual basis according to their progress due to past training (deVries, 1986). Finally, the user may produce if required a printed report of the athlete's file for the athlete.

\subsubsection{ANAEROBIC TEST - ANAEROBIC}

In Figure 5.1, the second component of the automatic data acquisition system is the anaerobic test. The equipment for this is described in 
Section 4.2.1. As with the software for the aerobic test the user completes the form that appears on the screen regarding the athlete's details. Again it is necessary to enter the athlete's surname and first initial. The source code listing for the anaerobic test software called ANAEROBIC is given in Appendix B.

The user can select to use automatic data collection from the cycle ergometer or to manually enter the data required. The data required are the total number of pedal revolutions over the duration of the test and the maximum and minimum pedal revolutions over any five second interval (Draper and Telford, 1989). If the automatic data entry option is chosen, the user is requested for the number of contrasting dots on the wheel. These black dots are spaced equally around the outer rim of the wheel. The diode detector detects the passage of each of these dots past the detector head.

For both the manual and automatic data entry options, the user enters the athlete's body weight and the load per kilogram of body weight into a screen form. The latter figure can be selected from an optimal load table displayed on the screen. This table, (see Appendix A.2) shows that the load is dependent on the level of training, the sex of the athlete and whether the arms or legs are being used for the test. The software calculates the recommended load rounded to the nearest 0.25 kilo pounds $(\mathrm{kP})$ to be set on the ergometer. This figure is rounded as it is difficult to refine the load to less than $0.25 \mathrm{kP}$. The user is allowed the option of using this recommended tension or of using another tension for reasons dependent on particular circumstances.

Prior to the commencement of automatic data collection, the user is asked to strike any key to select the gearing. This is done in the two or three seconds prior to the test while the athlete is pedalling the ergometer as fast as possible with no set load to overcome the inertia of the flywheel. The software continuously monitors the output of the detector and hardware and determines the time interval between changes in the polarity of the square wave. As outlined in Section 4.2.1, the square wave changes in polarity each time a pre 
defined number of contrasting spots has been detected. Initially the software instructs the hardware to change the polarity of the square wave at the detection of each spot. If the time interval between successive spots is less than one clock tick, the software instructs the hardware to change the polarity of the square wave at the detection of every second spot. This can be repeated with the software instructing the hardware to look at every fourth or eigth spot until the time interval between successive spots is one or more clock ticks. The instruction to the hardware is by a ROM BIOS call to send either a $0,1,2$ or 3 on the data pins to the printer port.

The clock ticks mentioned above refer to the PC's hardware clock ticks available through ROM BIOS which occur 18.2 times a second. This interrupt is provided by BIOS instructing the 8253 programmable timer chip to count 65,536 input pulses from the clock generator chip before generating an interrupt of its own (Sheppard, 1987). These interrupts form the basis of the DOS time of day services. For the purposes of this project it was found sufficient to use this resolution of approximately 1/20th second offered through the ROM BIOS. If in future the need arises for improved resolution caused by placing more spots on the ergometer wheel, it is possible to directly program the programmable timer chip to provide improved timing resolution (Sheppard, 1987).

Once the gearing is selected, the software instructs the user to strike any key to start the test. The software monitors the parallel port for the specified duration of the test. Each time the input square wave changes in polarity the number of clock ticks since the test started is requested through the ROM BIOS and this value is stored in memory.

In order to allow the software the maximum free time to monitior the parallel port during the test, the data are processed only at the conclusion of the test, to determine the total number of spots detected and the maximum and minimum number of spots detected in any five second period. The gearing and the ratio between the number of pedal revolutions to wheel revolutions are used to convert these 
three figures to the equivalent number of pedal revolutions. Using these values, the athlete's body weight and the set load, the software calculates the peak power, lowest power, total power, the peak power $/ \mathrm{kg}$, the lowest power $/ \mathrm{kg}$, the total power $/ \mathrm{kg}$, the relative power decline as a percentage and the absolute power decline in Watts/sec as in Draper and Telford (1989). The user has the option of saving these results onto the athlete's file on disc or of exiting from EXFIT. For the case of the athlete being tested for the first time, no file will exist and consequently one is created. The user has the option of producing a printed report of the athlete's results or of producing no report.

\subsubsection{TEST REPORT - PRINTREP}

This option in the main menu allows the printing of an athlete's past or present test results. It is designed to produce a written report of test results that the athlete may take to the coach. An example printout of the aerobic test results is provided in Figure 5.7. The source code listing called PRINTREP is given in Appendix $B$. The filename of the athlete's file may be entered or it may be selected from the displayed directory of any files with a. DAT extension on the data disc. The software now provides the user the option of no printout, printing the latest test results only, printing all the test results on the file or allowing the user to specify the number of past results to print. The software detects whether a printer is connected and on line so that the system does not crash if no printer is available. 
UNIVERSITY OF SOUTHERN QUEENSLAND

CENTRE FOR THE ASSESSMENT OF HUMAN PERFORMANCE

Assessment Report

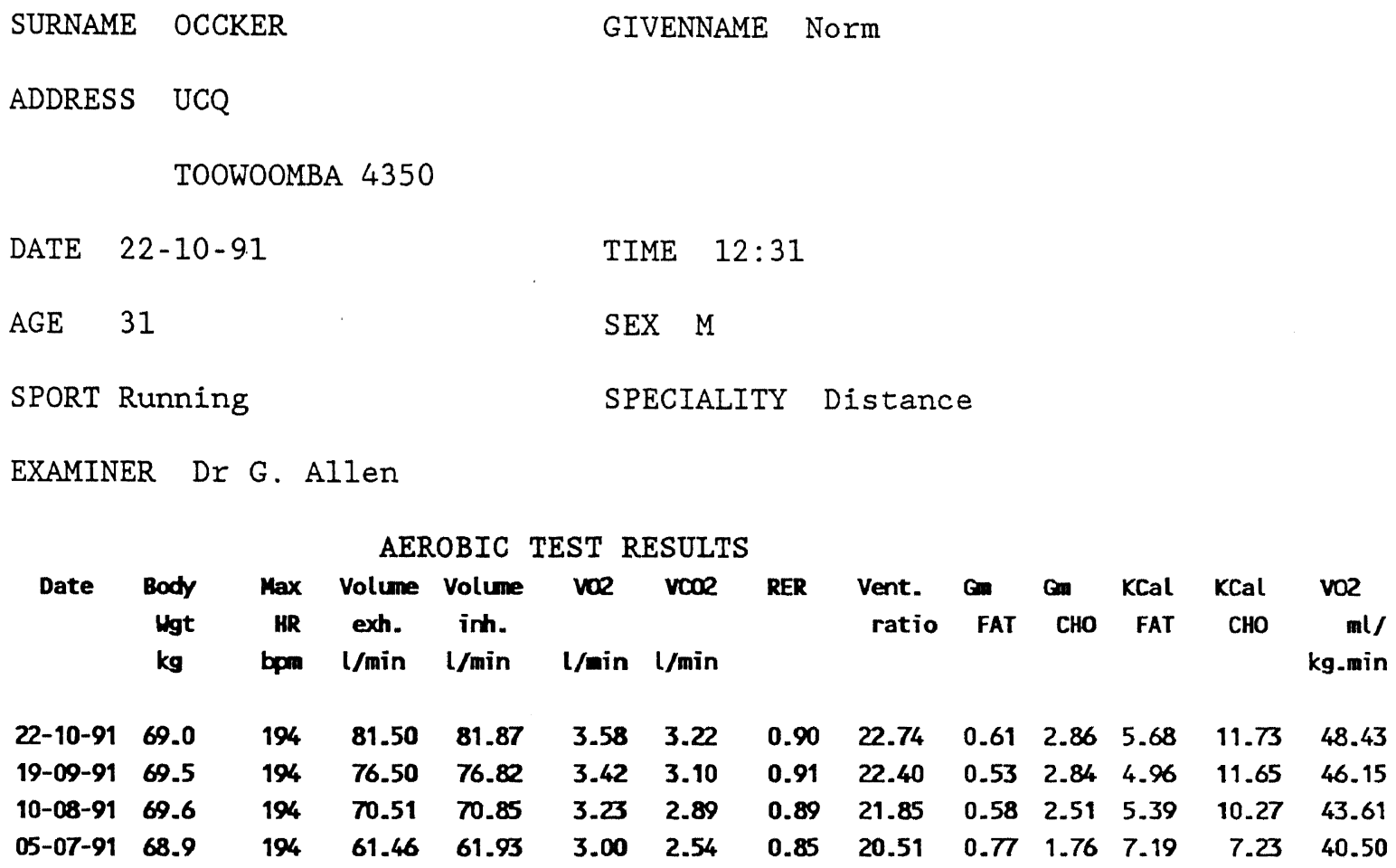

Figure 5.7 - Sample printout of the report of the aerobic test results 


\subsubsection{DISPLAY DATA RECORD - DISPLAY}

This option is designed to allow the user to view the records in the athlete's file on the screen. The source code listing called DISPLAY is given in Appendix B. As with the previous test report option, the user can enter the filename or select from the displayed directory. The records in the file are displayed on the screen using the FILESTR predicate in Turbo Prolog. This allows the user to move around any part of the screen by using the arrow, Home, End, PgUp and PgDn keys. Having viewed the records the user is allowed the option of deleting any unwanted or old records. The records to be deleted are selected from a menu. Each record selected is redisplayed on the screen and the user prompted as to whether to proceed with the deletion. 


\section{CHATER VI}

\section{EX P E R T S Y T E M}

\subsection{INTRODUCTION}

In essence, an expert system is a computer program or group of programs that encapsulate an expert's or several experts' skill within a particular field (Morrison and Forester, 1989). The expert systems in use today are designed to provide useful expertise in a single discipline or field of knowledge. The topical area is referred to as the domain of the expert system (Townsend, 1987). For EXFIT, the knowledge domain is fitness analysis. The expert in this case is the physiologist who is the Head of the Centre for the Assessment of Human Performance at the University of Southern Queensland. Gaps in the knowledge have been filled in by reference to textbooks on physiology. The expert system uses the domain knowledge and a series of appropriate inference procedures to make inferences in the field of knowledge that would be difficult enough to require significant expertise from a physiologist.

The application of expert systems in performance analysis has many potential benefits (Parisi and Allen, 1991a), including:

- EXFIT provides the interface between the physiologist and the coach and athlete. It provides the interface between the two disciplines of physiology and coaching. EXFIT does this by providing each athlete and the coach with a report containing an explanation of why particular aims and activities have to be followed. It explains to the coach and athlete in a logical and consistent fashion some of the science and physiology as to why particular activities have to be undertaken.

- The expert system provides an explanation of physiological changes that should be noted in the athlete undertaking the recommended activities.

- The associated software written with EXFIT provides for the aerobic and anaerobic power analyses a graph of the comparison of the athlete's performance with the average of other athletes in the same sport along with a graph of the athletes change in performance over testing sessions. Each of these graphs serve as 
providing motivation to the athlete.

- EXFIT can facilitate the training of students in physiology by comparing the student's response with the suggestions of the expert system.

\subsection{DESIGN OF THE ADVISORY SYSTEM}

The features which distinguish an expert system from an ordinary applications program is that the knowledge appears explicitly as a separate body in a knowledge base rather than appearing as part of the coding of the program (Ripple and Ulshoefer, 1987). This organization makes it much easier to encode and maintain the rules in the knowledge. As shown in Figure 6.1, this structure has been implemented in EXFIT. The remainder of this Chapter deals with the highlighted section of the Figure, namely the expert or advisory system.

In Figure 6.1, MAINTAIN is a Turbo Prolog program written to enable the domain expert to modify or add to the data and information. MAINTAIN takes into account the interrelationships between the data and information entries and so reduces the tedium and errors associated with manual updating. MAINTAIN is discussed further in the next Chapter. 
EXFIT: The Advisory System

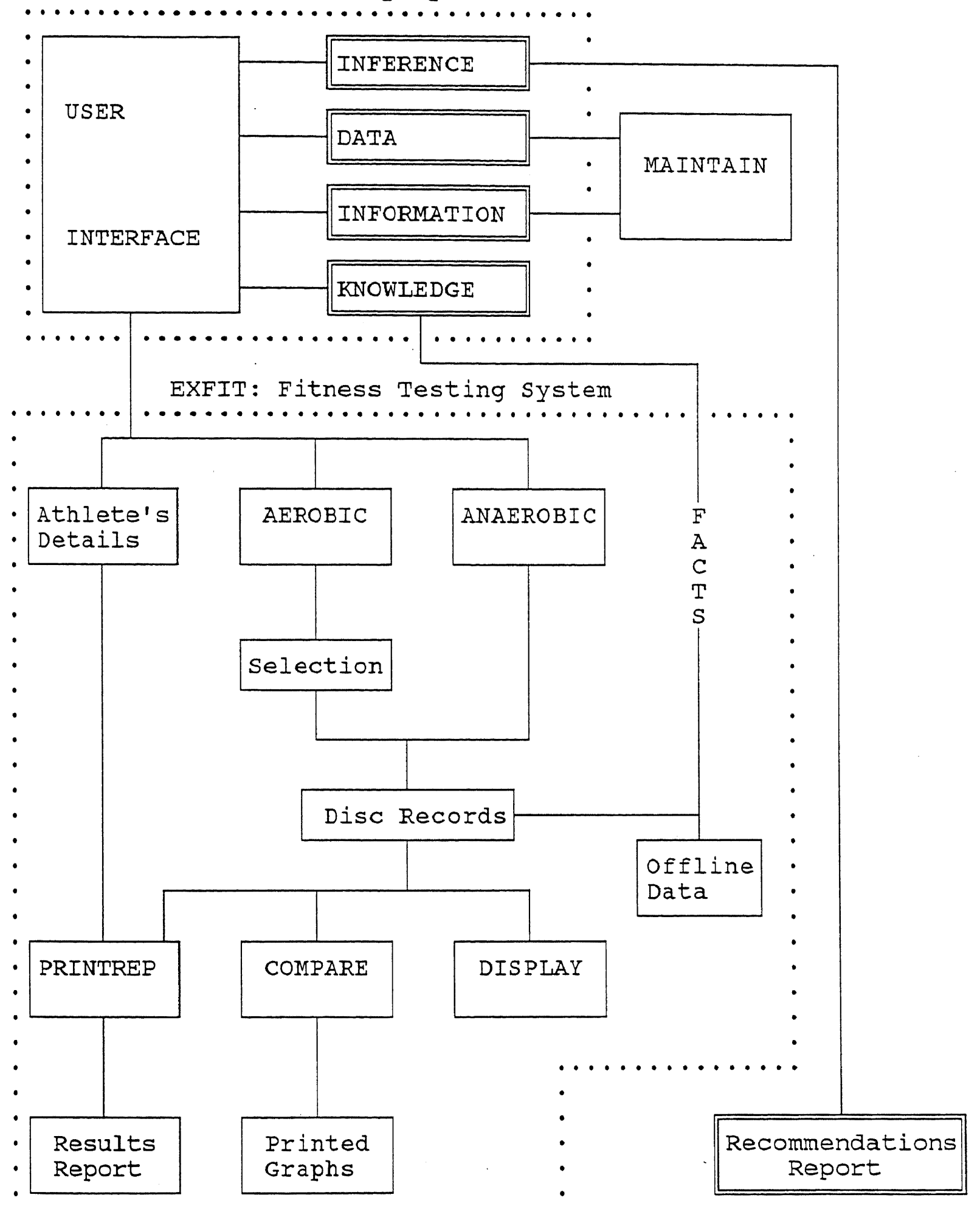

Figure 6.1 - The overall structure of the software of EXFIT 


\subsubsection{OVERALL STRUCTURE}

Typically, for example Liebowitz (1988), an expert system is described as consisting of three major components:

- User Interface

- Inference Engine

- Knowledge base

The user interface is the language interface by which the user accesses the expert system and so should be as user friendly as possible. This implies the need to identify the likely users of the system. The inference engine is a program that uses the facts supplied by the user and the rules in the knowledge base to deduce appropriate conclusions. The inference engine performs the dual tasks of inference and control (Townsend, 1987). The knowledge base comprises the expert's knowledge about the domain and consists of rules and facts.

Various 'standard' knowledge representations are listed in Jansen and Compton (1989), namely, production rules, frames, semantic nets, conceptual structures, inference networks and ripple down rules. Debenham (1989a) suggests that there are four common errors of knowledge representation. These are:

- The expert systems are not being designed to allow ease of maintenance. Areas of knowledge can overlap and be intertwined in other portions of knowledge. Often this is the result of the knowledge or rules being represented as the expert has pronounced them.

- The internal structure of the knowledge is not fully understood or incorrect assumptions are made about the structure by the knowledge engineer during the knowledge gathering process. This leads to the problems of recording incorrect knowledge and, secondly not necessarily recording all that the particular expert intended.

- Knowledge is being gathered and manipulated to conform to a particular expert system shell. If in the future there is a need to convert to a new shell, substantial conversion costs would be involved.

- The majority of expert systems are free standing and cannot interface with any corporate information processing resources such 
as database or spreadsheet programs.

These four errors give rise to four requirements during the design of an expert system. These requirements are repectively:

- A requirement to design the expert system for maintenance

- An understanding of the internal structure of the knowledge

- A representation of the knowledge in a form that is not committed to a particular expert system shell.

- A necessity to build expert systems that can be integrated with existing information processing resources.

The following Sections show how the EXFIT knowledge base has been designed to meet these requirements.

\subsubsection{KNOWLEDGE BASE - DATA, INFORMATION AND KNOWLEDGE}

In practice, the user interface, inference engine and knowledge base cannot be completely independent. The inference engine has to assume a certain structure of the knowledge. This leads to one of the problems mentioned above, namely that of maintainability. If a change is made to the structure of the knowledge base it may require a change to the inference engine. To minimize this, Debenham (1989a) suggests a splitting of the knowledge base into components containing data, information and knowledge. A great deal of effort was expended during this project to ensure that this separation occured in EXFIT. The structure used in EXFIT is outlined in Figure 6.1.

The terms "data", "information" and "knowledge" are consistent with the meanings given in Debenham (1989b), namely:

- The data are the actual real objects stored in the database. They are fundamental, indivisible real objects.

- Information is the relationships and associations between the items of data.

- The knowledge is the relationships between the items of information and/or the items of data.

The data and information are stored using the knowledge dictionary concept outlined in Jansen and Compton (1988), Jansen (1987) and Debenham (1985). In this representation, instead of using the 
conventional production rules in the form IF ... THEN ..., the data and information are decomposed into constituent components and stored as objects and the relationships between the objects in a conventional database. An example of the data in the knowledge base of EXFIT is:

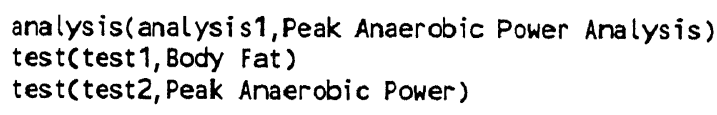

An example of the information is:

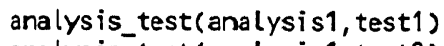

The implicit association is that in this case 'analysisl' consists of 'test1' and 'test2'. The data and information are stored in a normalized form as described in Jansen and Compton (1988). In this form each concept is fully defined and named once only. The aim and advantages of normalization are well known. The knowledge is represented by a group of Turbo Prolog clauses that relate the information and the data in determining in this example that the Peak Anaerobic Power analysis consists of a Peak Anaerobic Power test and a Body Fat test.

The following lists the set of Prolog clauses that relate the above data and information to provide a list, LIST2 containing the tests that have to be performed:

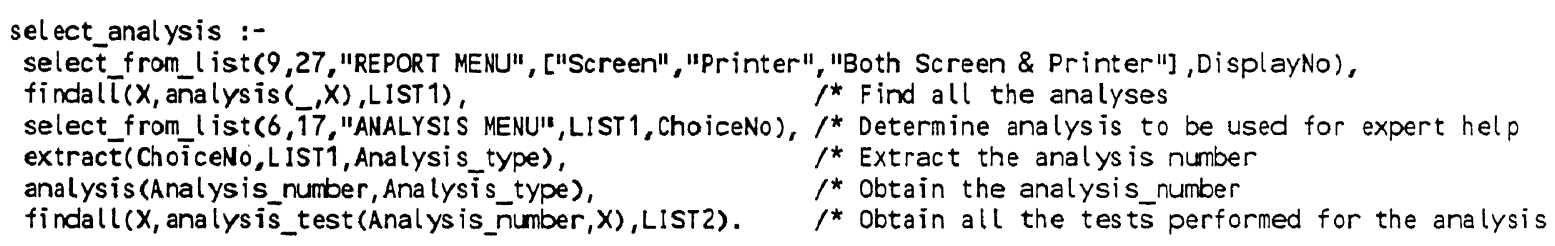

The tests extracted from the data and information are for the analysis selected by the user in the predicate 'select_from_list'.

The transfer of knowledge between the expert and the expert system is accomplished by a series of lengthy and intensive interviews between a knowledge engineer who is normally a computer specialist and a domain expert or experts who are able to articulate their expertise to some degree (Jackson, 1990). The knowledge in EXFIT has been obtained by a series of extensive interviews with the Head of the Centre for the Assessment of Human Performance. Gaps in the knowledge were filled by referring to authorative texts in the area. 
In order to determine the internal structure of the knowledge, several interviews with the domain expert were followed by the development of an initial prototype system and the use of this prototype to refine requirements and revise the prototype. Weitzel and Kerschberg (1989) outline this as the development life cycle of a knowledge based system. Debenham (1989a) suggests that a correct understanding of the internal structure of the knowledge helps to prevent recording knowledge incorrectly and to record all that the domain expert intends. The structure that is now used by EXFIT is shown in Figure 6.2.

The knowledge base of EXFIT consists of a series of analyses each consisting of a series of tests. The results of each series of tests allow the conclusion of a diagnosis. Each diagnosis has a series of aims necessary with the associated activities and follow up procedures. For each of the activities there is a series of explanations and warnings. 


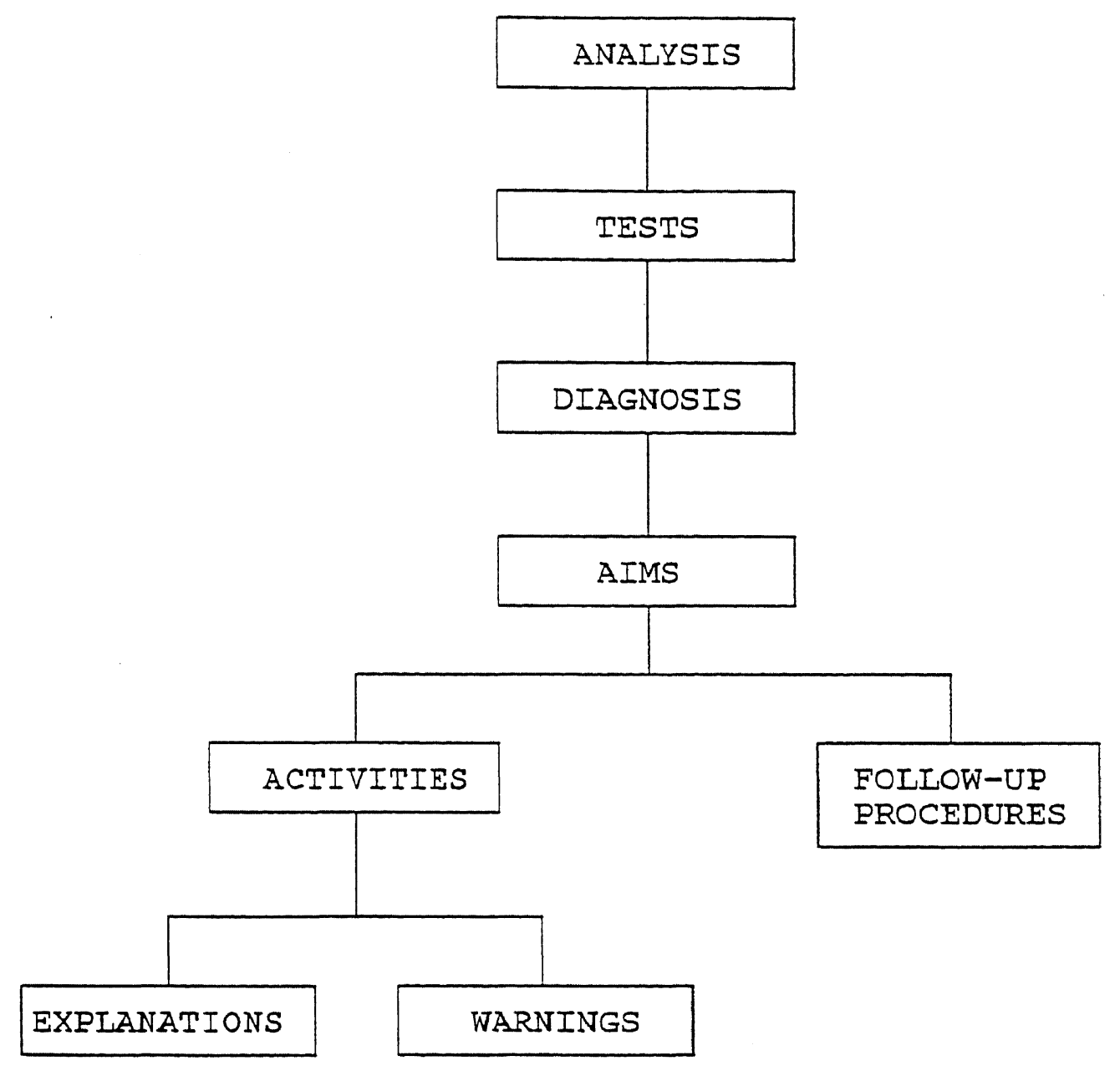

Figure 6.2 - Internal structure of the knowledge in the advisory system 


\subsubsection{INFERENCE}

The inference engine applies the facts that have been input into working memory to the knowledge base to infer a conclusion. Townsend (1987) outlines the four basic strategies of inference as forward chaining, backward chaining, heuristic reasoning and decision trees.

A chain, described by Giarratano and Riley (1989) is a succession of inferences connecting a problem with a solution. Forward chaining is a data driven process where the data are the starting point and the program works forward to determine if a conclusion is consistent with the facts. Forward chaining is effective in situations where the system has to interpret a set of incoming facts (Parsaye and Chignel1, 1988). This is the case in EXFIT where the incoming facts are the results from the tests. The alternative approach, not used in EXFIT, is the bottom up or backward chaining approach where the program starts from a possible conclusion and works backwards to determine if the conclusion is consistent with the facts available.

The analyses available in EXFIT are displayed in a screen menu from which the selection of the particular analysis to be carried out on the athlete is selected. Each particular analysis may require one or more tests to be performed. The tests that are to be carried out for each analysis are stored as part of the data and information and are determined by EXFIT following user selection of the appropriate analysis. The program now cycles through each test and for each one displays in a screen menu the possible results for that particular test. The possible test results for a test are stored as part of the data and information. For example:

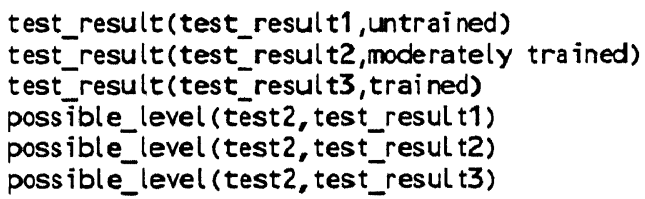

In this case EXFIT infers that 'test2' which has been previously determined to be the Peak Anaerobic Power test has the possible results 'untrained', 'moderately trained' and 'trained'.

The clauses that display the possible test results and allow 
selection of a result for each test are contained in the predicate 'actual_level':

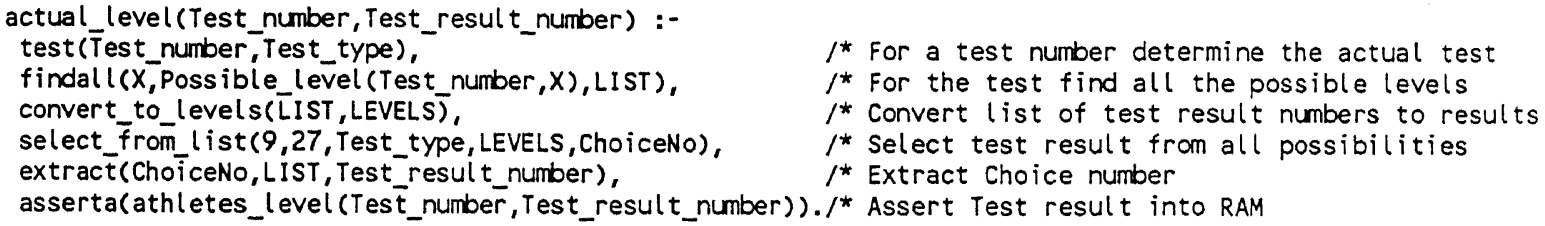

The last clause in the predicate asserts the results into RAM for later screen display and listing in the recommendations report of the tests and results used in producing the report. For each test, EXFIT uses a forward chaining search technique to conclude one or more possible diagnoses.

In EXFIT, as depicted in Figure 6.1, the facts are the results of the series of offline and online tests carried out on the athlete. These facts take the form of test results that have been processed either manually or automatically by the profiling system described in Section 5.3.3.1. This system profiles the athlete's test results with the means of the results of the other athletes in the database. For example, for the peak power test, this would indicate whether the athlete is at an 'untrained', 'moderately trained' or 'trained' level. The results from the profiling system provide the facts for EXFIT.

In EXFIT, the predicate 'find_possible_diagnosis' in the inference program scans the incoming facts from the results of the tests to determine a possible diagnosis. The following is a list of clauses in this predicate:

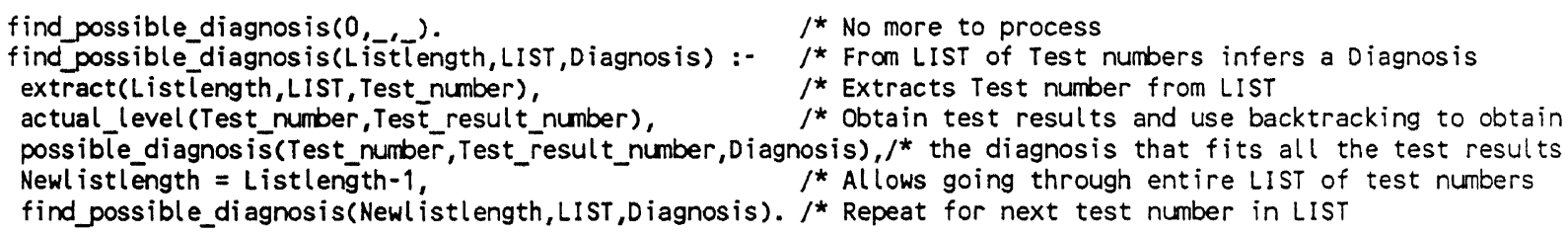

This predicate searches the possible diagnoses in the knowledge base in the order in which the clauses are listed. In Prolog, the search strategy is determined by the order of the clauses for the same predicate. An example of the 'possible_diagnosis' clauses are:

possible_diagnosis("test9", "test_result16", "diagnos is52"). possible_diagnosis("test10","test__resul t17", "diagnosis52"). possible_diagnosis("test11","test_resul t18","diagnosis52"). possible_diagnosis("test12","test_resul t17","diagnosis52"). 


\begin{abstract}
'Find_possible_diagnosis' obtains the test result for the tests in LIST and then backtracks through these clauses to conclude 'Diagnosis' which satisfies all the test results for the tests. EXFIT cycles through this predicate until the tests in LIST are exhausted.
\end{abstract}

Having obtained the diagnoses, EXFIT again searches the data and information to obtain one or more aims for the given diagnosis. The predicate 'find_aims' returns a list 'Aims_numbers' for 'Diagnosis' and at the same time lists the aims.

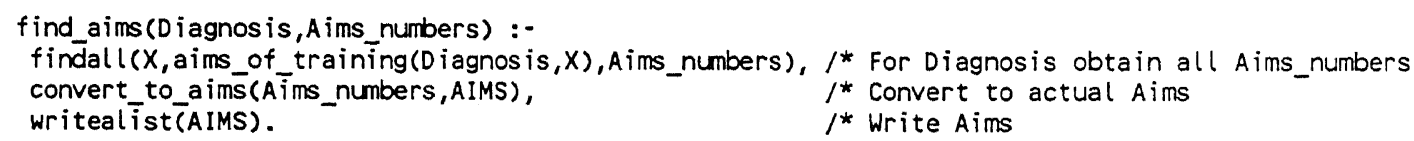

Similiarly for each aim a list of associated activities and follow up procedures are determined. For each activity, EXFIT performs another search to locate any explanations and warnings. Follow up procedures specify how long before the athlete should return for a retest to check for any improvements due to the recommended activities. Since one of the main purposes of testing is to monitor training effectiveness, it is apparent that the tests must be repeated following different phases of training. The explanations outline to the coach and athlete any physiological benefits that will occur due to the recommended activities. The warnings detail any particular point that must be observed. For example, for an anaerobic training program to increase alactic power output, a relevant warning is that the overload of muscles must be progressive throughout the program.

From Figure 6.2 and the above discussion on the inference process of EXFIT, it can be seen that the reasoning requires up to five stages in an inference chain. The stages consist of an inference of the tests required for the selected analysis followed by inference of the diagnosis, aims, activities and follow up procedures, explanations and warnings.

\title{
6.2.4 RECOMMENDATIONS REPORT
}

As each conclusion is obtained by EXFIT it is asserted into RAM using the dynamic database facilities of Turbo Prolog (Townsend, 1987). At the end of EXFIT's search for conclusions they are displayed on the screen or output to a printed report or both according to the user's 
requirements. An example output of the expert system (see Figure 6.3) provides a sample of the aims, activities, explanations, warnings and follow up procedures provided by EXFIT for the metabolic status of the muscle (aerobic capacity) analysis for the results shown in Figure 6.3 . 
AIM:

Increase glycogen level

Increase the level of aerobic markers in muscle

ACTIVITY :

Increase endurance training (intensity and duration)

Increase carbohydrate content of diet

Include 60 to 240 second surges of exercise in an

endurance training programme

EXPLANATION:

An increase in the stores of muscle glycogen can improve endurance performance. Muscle glycogen is a primary source of energy during prolonged aerobic activity Cardiorespiratory function will improve significantly Skeletal muscle will be affected in many ways by endurance training

(1) the ability to oxidise carbohydrates and fats will increase

(2) myoglobin concentration in the muscle will increase

(3) muscle stores of the fuels glycogen and triglycerides (energy sources) will increase

WARNING:

Do not increase your overall caloric load

FOLLOW UP PROCEDURE:

Return in six weeks

REASONING - test results used to provide recommendations are:

normal

moderately trained
Glycogen Level

Aer Markers - SDH, CS, CYT-OX

Figure 6.3 - Sample output of the expert system for the metabolic status of the muscle (aerobic capacity) analysis 


\subsection{CHOICE OF SOFTWARE PLATFORM}

In theory any computer language may be used to develop an expert system, however in practice procedural languages lead to unmaintainable systems. Smith (1988) states that in the construction of an expert system, it is necessary to:

- "set up a knowledge base consisting of facts and rules about a particular problem area"

- "be able to ask questions of the expert system without worrying about how the computer system will provide the answer."

In expert systems, the steps necessary for reaching a solution vary greatly. This indicates the utility of a declarative language which is supplied the necessary facts and rules by the knowledge engineer or user. The language system uses what it knows about the domain to define a procedure to arrive at conclusions (Townsend, 1987). This is the process that the human expert would call deductive reasoning.

Prolog, LISP and FORTH are appropriate languages for expert system development (Townsend, 1987). For this application, LISP and FORTH would have proved too low level with extensive memory requirements. The advantages of Prolog namely, that it is a high-level language and so more programmer friendly, that it has been implemented for use for IBM PC compatible computers and that a compiler was readily available made Prolog the obvious software choice.

An alternative considered was expert system shells. Shells contain specific strategies for knowledge representation, inference and control. To make the system ready for use, the user adds the knowledge for the domain. A problem with using shells is that the knowledge has to be twisted to suit the specific domains used by the particular expert system shell. This can reduce the generality of the system being developed. Moreover, the inference strategies and control available in a shell may or may not be appropriate to the purpose of the system.

The expert system and the associated maintenance program are written in Turbo Prolog version 2. Alternative versions of the Prolog language available at the time (1989) for the IBM PC were Arity 
Prolog and Prolog 2. An important factor in choosing Turbo Prolog was the availability of the Turbo Prolog Toolbox which is a collection of 80 -plus predicates. Liardet (1987) reviews the predicates in the Toolbox and concludes that they "fill an important gap with the extensive range of well-designed application utilities". Source code is provided for the Toolbox facilities written in Turbo Prolog itself. This proved useful for any occasional customisation that was required during the development of the expert system and the associated maintenance program. It is also informative just to see how other programmers tackle particular problems. When program development was started early in 1989, neither of the other two versions of Prolog offered as many facilities.

Turbo Prolog is not Edinburgh standard (Clocksin and Mellish, 1987), however the 'pure Prolog' aspects are essentially a subset of the standard. Non standard facilities, which vary from implementation to implementation have been included in a separate module and would require reprogramming if it is desired to port the expert system to another version of Prolog. 
CHATER VII

EXPERT SYSTEM MAINTENANCE

\subsection{Introduction}

The development process of an expert system inherently includes repeated validation and verification testing as part of the development process (Prerau, 1990). Each time the partially developed system was run to test the knowledge, all aspects of the program were tested as well to uncover any errors both in the knowledge and in the knowledge implementation.

Despite this, refinements and advances in knowledge require changes and updates to the rules of the expert system. Bryant (1988) indicates that the impetus for change originates from any of the following sources: the Expert, the knowledge base Engineer, the knowledge base Administrator or the User.

An important factor in the design of EXFIT was ease of maintainability. As outlined in the previous Chapter, this was done by having the user interface, data, information and knowledge as separate modules in EXFIT. In addition the design for maintenance was also achieved by normalizing the data and information or by storing them in their lowest possible element so that no parts overlap (Debenham, 1989a). If one part is modified there are no overlapping parts that also have to be modified. The module of EXFIT called MAINTAIN is designed to take into account the effect that any modifications or additions will have on the total system. This is important because the number of rules in a knowledge base grows exponentially with an increase in the number of parameters and all the possible values of these parameters (Simon et al., 1990). As the system grows beyond 50 or even 100 rules, the task of manually updating becomes complex due to firstly finding the rule that requires modification and secondly taking into account all the interrelationships that will have an effect on the total system. Consequently, an automated system that displays the current knowledge representation as it is being modified is required and MAINTAIN has been designed accordingly. 
Secondly, MAINTAIN provides a mechanism for assimilating the knowledge in a way that allows critical examination by the Expert. As outlined further in the next Section, the Expert is able to scan on the screen through all the available analyses in EXFIT with their associated tests and possible test results. On selection of a set of test results, the Expert can examine the diagnoses, aims, activities, follow-up procedures, explanations and warnings. MAINTAIN also allows the domain Expert to maintain, update and add to the rules of the data and information. The source code for MAINTAIN is provided in Appendix $B$ and is described in the next section.

\subsection{MAINTAIN}

MAINTAIN uses a series of menus and windows. The predicates from the Turbo Prolog Toolbox have been used to provide pop up menus and line menus. The source code of the predicate that uses the built in predicate MAKEWINDOW to produce a screen window has been customised so that the window number of the windows produced and the size of the windows in rows and columns is asserted into RAM. The reason for doing this is two fold:

- Firstly as the amount of window manipulation is large, it is necessary to have a means of keeping track of the windows displayed and to clear them on completion.

- Secondly at a point in the software the tests displayed in the test window have to be read. This is done using the field_str built in predicate which reads the specified number of columns in a row. This is repeated for the number of rows in a window and so for each test window the number of rows and columns in the window are required.

The software of MAINTAIN is written along the lines of EXFIT with a set of predicates that form the user interface and are independent of the structure of the data and information.

It has been assumed that only more experienced users will utilize MAINTAIN. Nevertheless the principles outlined in the next Chapter on user interface have been adhered to. 
Upon entry to the program the first line menu encountered is as follows :

Consult file 1st scr 2nd scr Save Quit

For all line menus in the MAINTAIN program, the user employs the left and right arrow keys or employs a key letter to select a choice. Once selected the appropriate choice is highlighted and execution continues.

Further manipulations are dependent on the data and information files being loaded into memory using the "Consult file" option. The "lst scr" option displays and allows modifications to the analyses, tests and test results only. The rationale for limiting the first screen to this is that the screen does not become overcluttered and the user is not flooded with information (Galitz, 1989). In the "2nd scr" option, no changes $c$ an be made to the analyses, tests and test results. However the selected analysis and appropriate tests and test results are displayed so that the user does not have to remember data from one screen to another. Patkin (1987) emphasises that it is important to reduce the demands on short term memory. Modifications are made to the diagnoses, aims, activities, follow up procedures and warnings. "Save" allows the user to save the data and information files by overwriting the original files or by saving in new files. The READFILENAME predicate from the Turbo Prolog Toolbox is used in determining the filenames. "Quit" returns the user to DOS as does pressing the $\langle$ Esc $\rangle$ key. This is to follow the consistency aim of the interface design.

A sample of the first screen is shown in Figure 7.1. The line menu options have been designed to perform the following functions. "Display" allows selection of the next analysis for which to display the tests and test results. The tests and test results displayed apply only to the selected analysis. The selected analys is remains highlighted in the window. In this case it is the $\max \mathrm{VO}_{2}$ analysis. Only three analyses appear in the analyses window at any time. Again this is to minimise the amount of information on the screen. The LONGMENU predicate in the Turbo Prolog Toolbox has been used to allow the user to scroll through the entire list of analyses. 


\begin{abstract}
"Next" and "Previous" allow movement from window to window to select the window on which the "Add", "Modify" or "Remove" option will be used. The window currently being considered overwrites any other windows and becomes highlighted. "Add" allows the addition of a new entry in the selected window. "Modify" enables the modification of the selected entry in a window. "Remove", deletes the selected entry from the data and information. Following each "Add", "Modify" or "Remove", the screen is automatically updated and the updated rule is asserted into RAM and the old rule retracted. The "Exit" option returns control to the initial main menu. The option to return to the main menu is also available by pressing the $\langle$ Esc $\rangle$ key.
\end{abstract}




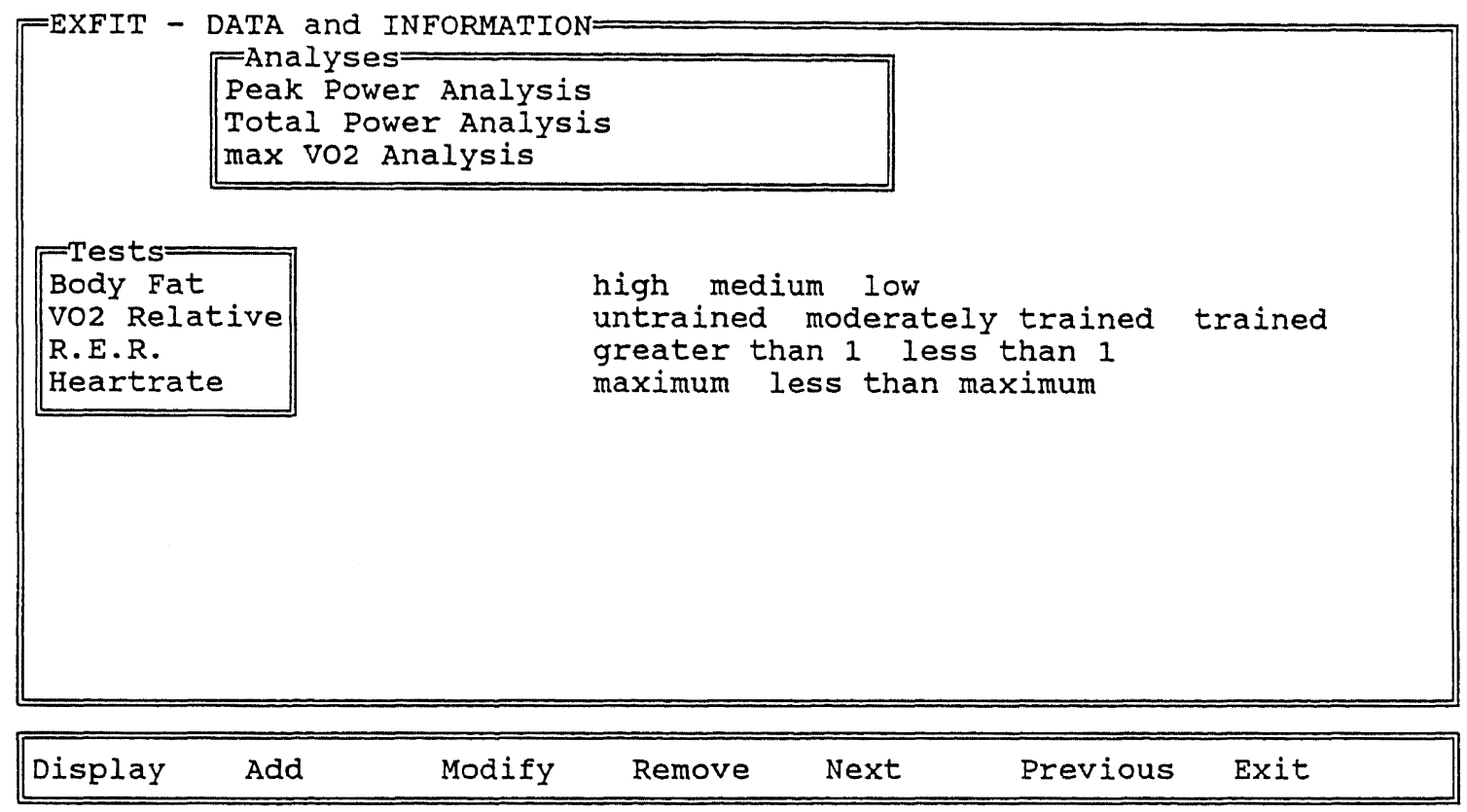

Figure 7.1 - Sample of the first screen in MAINTAIN 
Figure 7.2 provides a sample of the second screen. The options in the line menu have the same functions as for the first screen. For the selected analysis, tests and test results the appropriate diagnosis, aims, activities, follow up procedures and warnings are provided, each in a window of its own. The size of each window is automatically adjusted to the maximum width of the screen so that each entry fits within it. The amount of information on the screen in this case is too much to use tiled windows where the contents of each window are always visible. Using the guidelines in Galitz (1989), overlapping windows are suitable in situations where window manipulation can be tolerated and where the user has a certain level of expertise. Both these assumptions are valid in the case of MAINTAIN. The "Next" and "Previous" options are used to move between windows. As this is done each window in turn becomes highlighted and fully visible. 


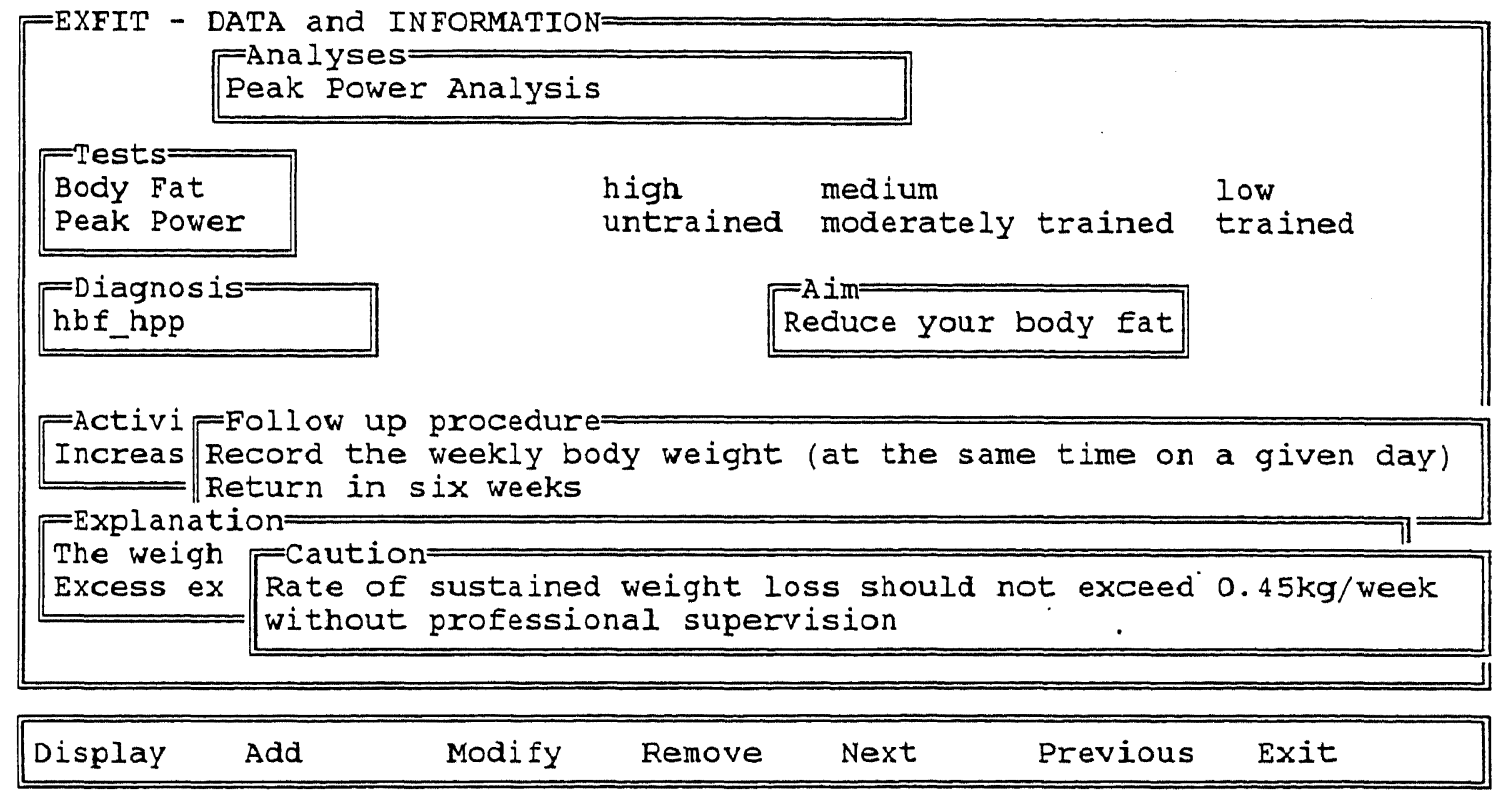

Figure 7.2 - Sample of the second screen in MAINTAIN 
Using the structure of the knowledge in Figure 6.2 in the previous chapter it is seen that each diagnosis could have one or more aims which in turn could have one or more activities and follow up procedures. Each of the activities could have one or more explanations and warnings. There is insufficient space on the screen to display every possible case. This is overcome by providing for each window the possibility of checking if there is more than one piece of information for each window by using the "Next" and "Previous" options to move to the appropiate window and then selecting the "Display" option. For example in Figure 7.2 , if the activities window is highlighted and the "Display" option used, a second activity and associated explanation and warning are displayed for the aim. Similiarly, for the activity in Figure 7.2, by highlighting the explanation window and selecting the "Display" option, if there is a second explanation and associated warning for the explanation it is displayed. "Add", "Modify" and "Replace" may now be applied to the updated display as required. If there are no more cases to display, the screen display remains unchanged. In this fashion all possible entries of the data and information may be displayed and updated as required. 


\section{CHA P T R VIII \\ USER INTERFACE}

\subsection{Design}

Every interactive computer software system has a user computer interface. If this user interface is friendly and easy to use, then the potential users will be encouraged to use the system (Price, 1990). In EXFIT, the user interface is designed to fulfill two aims, namely:

- the user interface is easy to use and as user friendly as possible.

- as outlined in section 6.2.2, the user interface is totally independent of the data, information and knowledge. A change to any of these three will not require a change to be made to the user interface.

of the various user interface styles that exist such as question and answer, menu selection, form fill-in, command language, natural language and direct manipulation (icons), (Galitz, 1989) menu selection and form fill-in have been implemented to achieve the first aim.

To achieve the second aim, the predicates in the user interface have been structured to be independent of the knowledge base. The predicates for producing windows, forms, menus and for list handling, display and printing are grouped within the user interface. A series of lists and files are used for communication in both directions between the knowledge base and user interface with the predicates in the user interface structured to be independent of the contents of these lists and files.

\subsection{Implementation}

Figure 8.1 provides a schematic of a sample of the interaction of the user with the system. The progress from screen to screen for the expert advice or advisory system and for the aerobic test is depicted. As illustrated in the Figure, all control starts from and returns to the main EXFIT menu. In EXFIT, both single choice and multiple choice menus are used. Galitz (1989) outlines that menu 
screens are effective due to the exploitation of the more powerful capability of humans to recognize rather than recall. A selection is made by using the arrow keys to move the highlight to the required choice and pressing the <Enter> key. Also a choice may be made by pressing the key letter of the selection. With the multi choice menus, any highlighted selection may be changed by pressing <Enter> again on the selection and it is deselected and the highlight removed. 


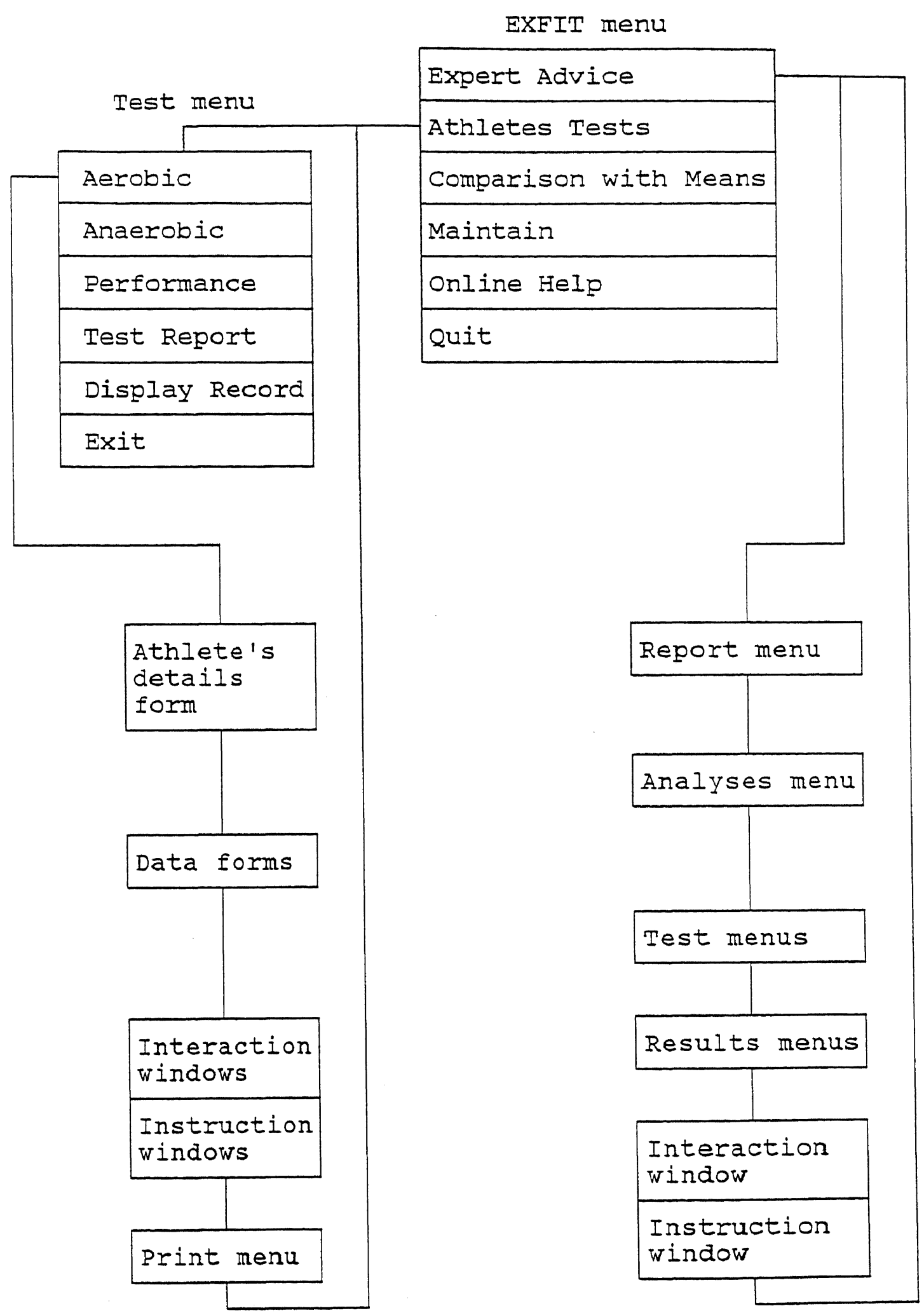

Figure 8.I - Example of the user interface's flow from screen to 
In Figure 8.1, form filling has been used for entering the athlete's personal details and for any manual data input related to the aerobic and anaerobic tests. This method has been utilized for this input because it provides the user with the cues necessary to determine easily what must be keyed along with a degree of control over what is happening by allowing the user to move between fields, modify data that have been entered, view the completed form before continuing and providing a limited amount of checking of the input fields. Any keystrokes that are not acceptable are ignored and an alarm beep sounded. Editing of the form may be done at anytime by using the arrow keys to move to the field to be edited. <Enter> also causes the cursor to move to the next field in the form. On completion, the form is previewed for any errors and if correct is saved to disc by pressing the $<F 10>$ key.

Consistency is a cardinal rule in the design of any user interface (Galitz, 1989). Consistency reduces the amount of learning by the user operating the system, but at the same time as providing consistency, it is necessary to capture the unique and particular features of different tasks (Chignell and Waterworth, 1991). Throughout EXFIT, consistent screen formats and keystrokes have been implemented. Each screen has the same format with a window that fills the majority of the screen for the user interaction and an instruction window of constant shape and colour at the bottom of the screen. Each screen is titled to provide the user with some concept of the usage of the window. Consistency is also vital between menus (Galitz, 1989). In EXFIT, choices such as Exit which are found on more than one menu are positioned consistently on all menus.

Consistency of keystrokes has also been implemented, namely: $<E S C\rangle$ to exit from menus and screens; $\langle$ F10 $>$ to complete the choice from multi choice menus and to complete entry into a screen form; $\langle F 1\rangle$ to provide context sensitive help at the stages of the consultation where it is available; $\langle$ F10 $\rangle$ to obtain a printout of a graph on the screen and to change the time frame interval during the aerobic test.

New users of a computer software system must undergo a learning 
process and Galitz (1989) points out that on-line help that supplements hard copy documentation is necessary. In EXFIT, a help system is available by selecting the "On Line Help" option in the main menu. This Help information is stored in a standard text file and is displayed in the user interaction window. The information fills more than one screen and the PgDn and PgUp keys are used to access all the information. Pressing the $\langle E S C\rangle$ key exits from the help screen. In addition, a context sensitive help facility is available at crucial stages of the expert system consultation. Pressing the $\langle F 1\rangle$ key provides this help. To supplement these on-line help facilities, a more detailed description on the use of the programs is available in a Users Manual which is shown in Appendix $C$. 


\section{TESTS AND RESULTS}

\subsection{Expert System}

Following the development of the recommendations of EXFIT by the cyclic process outlined in section 6.2.2, a sample of eight recommendations were submitted to four professional coaches, namely a basketball, a cycling and two swimming coaches for comments. The sample of recommendations provided to the coaches are attached in Appendix D. Each recommendation had a feedback sheet attached to it with questions and a space for comments. At the time of writing, one coach had not responded. A summary of the responses from the other three coaches to the questions are provided in Table 1. A summary of the comments is provided in Table 2 . In general the recommendations were well received with positive feedback. From the comments of the coaches, any of the recommendations of EXFIT that required enlarging on have been modified and expanded as suggested.

Although further testing of the system was planned, the failure of the carbon dioxide analyser and the elapse of five months before final repair prevented complete testing before the submission of this thesis. Nevertheless, the system will be subject to extensive review and further development in the future. As outlined in Chapter 8 , the module MAINTAIN allows ease of update and modification to the knowledge by the domain Expert. The domain Expert may modify any of the analyses, tests, test results, diagnoses, aims, activities, follow up procedures, explanations and warnings without the presence of the knowledge engineer. 


\begin{tabular}{|c|c|c|c|c|c|c|c|c|}
\hline & \multicolumn{8}{|c|}{ Recommendation number } \\
\hline $\begin{array}{l}\text { Recommendations are } \\
\text { useful }\end{array}$ & $3 Y$ & $3 Y$ & $3 Y$ & $3 Y$ & $3 Y$ & $3 Y$ & $3 Y$ & $\begin{array}{l}2 Y \\
1 \mathrm{M}\end{array}$ \\
\hline $\begin{array}{l}\text { Recommendations are } \\
\text { comprehensive }\end{array}$ & $\begin{array}{l}2 Y \\
1 M\end{array}$ & $\begin{array}{l}2 Y \\
1 M\end{array}$ & $\begin{array}{l}2 Y \\
1 N\end{array}$ & $\begin{array}{l}2 \mathrm{Y} \\
1 \mathrm{M}\end{array}$ & $\begin{array}{l}2 Y \\
1 M\end{array}$ & $3 Y$ & $\begin{array}{l}2 Y \\
1 M\end{array}$ & $\begin{array}{l}2 Y \\
1 M\end{array}$ \\
\hline $\begin{array}{l}\text { Recommendations are } \\
\text { understandable }\end{array}$ & $3 Y$ & $3 Y$ & $3 Y$ & $\begin{array}{l}2 Y \\
1 M\end{array}$ & $3 Y$ & $3 Y$ & $\begin{array}{l}2 Y \\
1 M\end{array}$ & $\begin{array}{l}2 Y \\
1 \mathrm{M}\end{array}$ \\
\hline $\begin{array}{l}\text { Activities are } \\
\text { appropriate }\end{array}$ & $3 Y$ & $\begin{array}{l}1 Y \\
2 \mathrm{M}\end{array}$ & $3 Y$ & $\begin{array}{l}2 \mathrm{Y} \\
1 \mathrm{M}\end{array}$ & $3 Y$ & $\begin{array}{l}2 Y \\
1 M\end{array}$ & $3 Y$ & $\begin{array}{l}2 \mathrm{Y} \\
1 \mathrm{M}\end{array}$ \\
\hline $\begin{array}{l}\text { Explanations are } \\
\text { helpful }\end{array}$ & $\begin{array}{l}1 Y \\
2 M\end{array}$ & $\begin{array}{l}2 Y \\
1 M\end{array}$ & $\begin{array}{l}1 Y \\
2 M\end{array}$ & $\begin{array}{l}2 Y \\
1 \mathrm{M}\end{array}$ & $\begin{array}{l}2 Y \\
1 M\end{array}$ & $3 Y$ & $\begin{array}{l}2 Y \\
1 M\end{array}$ & $\begin{array}{l}2 Y \\
I M\end{array}$ \\
\hline Warnings are useful & $2 \mathrm{M}$ & $3 Y$ & $\begin{array}{l}2 Y \\
1 \mathrm{M}\end{array}$ & $\begin{array}{l}2 Y \\
1 N\end{array}$ & $\begin{array}{l}2 Y \\
1 M\end{array}$ & $3 Y$ & $2 Y$ & $\begin{array}{l}2 Y \\
1 M\end{array}$ \\
\hline $\begin{array}{l}\text { Recommendations can be } \\
\text { used in coaching an athlete }\end{array}$ & $3 \mathrm{M}$ & $\begin{array}{l}1 Y \\
1 M\end{array}$ & $3 Y$ & $3 Y$ & $3 Y$ & $\begin{array}{l}2 Y \\
1 M\end{array}$ & $2 Y$ & $\begin{array}{l}2 Y \\
1 M\end{array}$ \\
\hline
\end{tabular}

Y - Yes, M - Maybe, N - No

Table 1 - Summary of responses from the coaches 
Recommendation 1

- All phases are relevant to my training squad. Your recommendations are very helpful and will be utilized throughout this current season

Recommendation 2

- The aims-targets-goals are excellent. I have never been a great believer in the use of weights and lean towards isokinetic and isotonic exercises to improve muscular strength in all young swimmers. I believe and appreciate your advice relating to anaerobic power output and I will give it special thought for 1991-92.

- More comments on warnings for exercising the larger muscle groups

Recommendation 3

- Your recommendations meet with my complete approval

- Explanation and warning OK. May need more explanation as the weight loss can vary because of normal training activity

Recommendation 4

- I do not fully understand the suggested activity. This is only through my ignorance of scientific testing. Your explanation is very specific and very helpful.

- More explanation with warnings

Recommendation 5

- Excellent!! I heartily agree

Recommendation 6

- I would like to learn a lot more about increasing iron level and increasing iron intake. Your explanation regarding dietary source is excellent

Recommendation 7

- These procedures are followed by me in the course of training

- More explanation on recovery period

Recommendation 8

- Excellent

Overall comment

- Generally it was well presented in a way that most coaches would find easy and inviting to use.

Table 2 - Summary of comments from the coaches 


\subsection{On-Line Data Acquisition}

The software of EXFIT for the data acquisition system of the aerobic test has been extensively tested and is currently used at the Centre for the Assessment of Human Performance. The aerobic test has been run in conjuction with the manual data collection system and the two sets of results compared. The results have agreed within the inherent error levels of the equipment. Additionally, the data acquisition system software has been run to test a series of subjects as part of the work for a Biology Honours student's project, titled, "Factors Affecting Aerobic Recovery from Exercise". Any suggestions that have arisen during these tests have been implemented. For this project, the system is being used for testing the subjects under both maximal and submaximal workloads and during recovery and rest periods over a minimal period of four hours requiring continual use of the system, together with utilisation of the option to vary sampling intervals.

The system has also been used to monitor athletes during maximal and submaximal tests on either the cycle ergometer or the treadmill. The value of the on-line data acquisition system has been demonstrated in these tests. The system provides much greater control and allows the necessary data values to be recorded only at the point at which the athlete is exercising at maximum workload.

The automatic data acquisition system for the anaerobic test has been tested by running the system in parallel with the manual data collection system during the testing of an athlete. The two sets of results agreed and given the higher resolution of the automatic data acquisition system, it provides results with a higher degree of accuracy. 


\section{CHA P T R X \\ CONCLUSIONS}

\subsection{Conclusion}

An expert system applied to the area of fitness analysis has been developed. This intelligent front end provides an advisory system for the results from both an automatic fitness testing system and from an off-line system. The usefulness of expert system technology has been proven in a new field. The motivation to build the expert system was to improve the feedback from the results of physiological assessments of the athlete to the coaches. EXFIT has been specifically designed to provide recommendations to do this in a logical and systematic report to the coach and athlete.

In EXFIT the domain specific knowledge of the physiologist has been codified and the expert system uses this knowledge to provide recommendations. An effort has been made to recognize the structure of the knowledge and the internal knowledge representation in EXFIT uses this structure. The knowledge and knowledge structure have been tested by an iterative process which took the form of a series of lengthy interviews with the domain expert, followed by the production of a prototype along with a return to the domain expert for testing of this prototype as a consequence of which there were further interviews required with the domain expert to further develop and refine the prototype. This process was repeated to obtain the package as it now exists. Finally a sample of recommendations were provided to four professional coaches for their comments which have been incorporated into the recommendations.

The knowledge is represented in a form that is not committed to a particular expert system shell. This has been achieved by separating the knowledge from, and ensuring it is completely independent of the user interface. MAINTAIN, the program for updating the expert system has also been designed to separate the predicates for the user interface from the predicates that are knowledge related.

As EXFIT will be subject to extensive review and further development, 
the system has been designed to accommodate both future expansion and changes and advances in knowledge. Consequently it was vital for EXFIT to be designed to allow ease of maintenance. This has been done by splitting the knowledge base into the separate modules of data, information and knowledge with the user interface as another seperate module. The data and information have been stored using a knowledge dictionary format which presents a methodology for not merely codifying the data and information but for representation in a form whereby any maintenance and updating can be carried out systematically. In the knowledge dictionary, the data and information have been normalized or stored in the lowest possible element so that no parts overlap. If one part is modified then there are no overlapping parts that also have to be modified.

In this fashion, the design of the knowledge base provides a thorough, practical testing of the theory of knowledge base design proposed by Debenham (1989a) whereby the expert system is designed for maintenance, the knowledge was gathered with regard to it's internal structure, the knowledge is represented as being independent of a particular expert system shell and the expert system is integrated with the information processing resources in the Centre for the Assessment of Human Performance, namely the on-line data acquisition system.

This computer controlled data acquisition system was developed for the aerobic power and the anaerobic (peak and total) power to provide results for the expert system. This system provides automatic data acquisition, displays, files and prints the results, plots the athletes change in performance with time and compares the athlete's results to the averages of other athlete's results. This system is currently being utilized in the physiological testing of athletes and non athletes.

The expert system and maintenance program are written in Turbo Prolog 2.0. The programs written for the automatic data acquisition have been written using Turbo Pascal 4.0. Turbo Prolog 2.0 was found to be programmer friendly with the trace or debug facility proving to be 
invaluable. In addition the predicates for screen and menu handing and form setup within the Turbo Prolog Toolbox proved to be an excellent collection during the programming of the user interface. The programs for the automatic data collection make extensive usage of file handling, access to the operating system, calculations and graphics. Turbo Pascal 4.0 proved adequate for the implementation of these features.

\subsection{Further Developments}

The expert system will be reviewed and developed according to any additional requirements and user requests. The knowledge will undergo continual refinements. Additionally the number of analyses with their associated tests, diagnoses, aims, activities, explanations, warnings and follow up procedures will be increased as part of the expansion of the system. In conjunction with this, several developments that may be made as funding becomes available are to:

- Store on the athletes disc report, the results of the athlete"s tests from the off-line analyses.

- Automatically sample the heartrate and body temperature of the athlete via the $A / D$ converter

- Sample the barometric pressure and ambient temperature through the A/D converter

- Integrate the recommendations of the expert system into the disc report

- Upon the entry of the athlete's surname and initial, recall the personal details of an athlete who has been previously tested

- Selection by the software of the records at which the athlete is exercising at maximum workload during the $\mathrm{vO}_{2}$ max analysis. 
AITKENS, J.S., KUNZ, J.C., SHORTLIFFE, E.H., (1983), PUFF: An Expert System for Interpretation of Pulmonary Function Data, Computers and Biomedical Research, Vol 16, pp 199-208.

BANKS, W., (1988), Turbo Pascal 4.0, Byte, February 1988, pp 153-156.

BECKMAN INSTRUMENTS INC., Manual for Beckman LB-2 and OM-11 Gas Analysers, 2500 Harbor Boulevard, Fullerton, CA 92634, USA.

BORLAND INTERNATIONAL INC., (1987a), Turbo Pascal Owner's Manual, 4585 Scotts Valley Drive, Scotts Valley, CA 95066, USA.

BORLAND INTERNATIONAL INC., (1987b), Turbo Prolog Toolbox Users Guide $\notin$ Reference Manual, 4585 Scotts Valley Drive, Scotts Valley, CA 95066 , USA.

BORLAND INTERNATIONAL INC., (1988a), Turbo Prolog 2.0 Users Guide, 4585 Scotts Valley Drive, Scotts Valley, CA 95066, USA.

BORLAND INTERNATIONAL INC., (1988b), Turbo Prolog 2.0 Reference Manual, 4585 Scotts Valley Drive, Scotts Valley, CA 95066, USA.

BRIDGER, M., \& GORESKY, M., (1985), High-Resolution Printer Graphics, Byte, November 1985, pp 219-232.

BROOKS, G. A., \& FAHEY, T. D., (1985), Exercise Physiology, Macmillan Publishing Co., New York.

BRYANT, N., (1988), Managing Expert Systems, John Wiley \& Sons, New York.

CHIGNELL, M.H., \& WATERWORTH, J.A., (1991), Whimps and Nerds: An Extended View of the User Interface, SIGCHI Bulletin, Vol 23, No 2 , pp 15-21.

CLOCKSIN, W. F., \& MELLISH, C. S., (1987), Programming in Prolog, 3rd ed, Springer-Verlag, New York.

DALE, N., \& WEEMS, C., (1987), Pascal, 2nd ed, D. C. Heath \& Co., Lexington.

DANZ INSTRUMENT SERVICE, (1989), Manual for Triple Port Valve Controller, 84 Brighton Parade, Blackwood, SA, 5051.

DEBENHAM, J. K., (1989a), Managing Expert Systems: Four Key Issues, in Proceedings of the Fifth Australian Conference on the Applications of Expert Systems, Sydney, pp 163-188.

DEBENHAM, J. K., (1989b), Knowledge Systems Design, Prentice Hall, New York. 
deVRIES, H. A., (1986), Physiology of Exercise, 4th ed, WCB Group, Dubuque.

DRAPER, J., \& TELFORD, R., (1989), Sport Specific Guidelines for the Physiological Assessment of the Elite Athlete, National Sports Research Program, Canberra.

FAGAN, L.M., KUNZ, J.C., FEIGENBAUM, E.A., OSBORN, J.C., (1984), Extensions to the Rule-based Formalism for a Monitoring Task. In B. Buchanan and E. Shortliffe (eds), "Rule Based Expert Systems", Addison-Wesley, Reading, Mass., pp 397-423.

FOX, E. L., \& MATTHEWS, D. K., (1981), The Physiological Basis of Physical Education and Athletics, CBS College Publishing, Philadelphia.

GALITZ, W., (1989), Handbook of Screen Format Design, 3rd ed, QED Information Sciences, Inc., Wellesley.

GASTON, L.W., LINDBERG, D.A.B., VANKER, A.D., KINGSLAND, L., (1983), AI/COAG, A Knowledge-based Surrogate for the Human Hemostasis Expert, Vol 80, pp 185-188.

GIARRATANO, J., \& RILEY, G., (1989), Expert Systems $=$ Principles and Programming, PWS-Kent, Boston.

HORN, H.A., COMPTON, P., LAZARUS, L., QUINLAN, R., (1985), An Expert System for the Interpretation of Thyroid Assays in a Clinical Laboratory, The Australian Computer Journal, Vol 17, No 1.

HAWKE, A.K., MARLIN, C.D., (1988), Expert Systems in Crisis Management: Ambulance Dispatch, The Australian Computer Journal, Vol 20, No 1, pp 1-8.

JACKSON, P., (1990), Introduction to Expert Systems, 2nd ed, AddisonWesley, Wokingham.

JANSEN, B., \& COMPTON, P., (1989), The Knowledge Dictionary: Storing Different Knowledge Representations, in Proceedings of the Fifth Australian Conference on the Applications of Expert Systems, Sydney, pp 143-162.

KRUSE, R. L., (1987), Data Structures and Program Design, 2nd ed, Prentice Hall, New Jersey.

LAMB, D. R., (1984), Physiology of Exercise, 2nd ed, Macmillan Publishing Company, New York.

LIARDET, M., (1987), Turbo Prolog Toolbox, APC, August 1987, pp 99107.

LIEBOWITZ, J., (1988), Introduction to Expert Systems, Mitchell Publishing Inc., New York. 
MacDOUGALL, J. D., WENGER, H. A. \& GREEN, H. J., (1982), Physiological Testing of the Elite Athlete, Mouvement Publications Inc., New York.

MILLER, R.A., POPLE, H.E., MYERS, J.D., (1982), INTERNIST-1: An Experimental Computer Based Diagnostic Consultant for General Internal Medicine, New England Journal of Medicine, Vol 307, No 8, pp 468-476.

MORGAN INC., (1988a), Manual for PKC1 Data Acquisition Card, 2 Dundee Park Level One, Andover, Massachusetts.

MORGAN INC., (1988b), Manual for Ventilometer Mark 2, 10 Manor Road, Chatham, Kent.

MORRISON, P., \& FORESTER, T., (1989), Words of Doubtful Wisdom, The Australian, 3rd Oct, 1989.

NAGLE, F. J., (1973), Physiological Assessment of Maximal Performance, Exercise and Sports Sciences Reviews, Vol 1, pp 313338.

NORTON, P., (1986), Inside the IBM PC, Prentice Hall Press, New York.

OWENS, R., (1985), FITSCAN: A Computer Monitoring and Analys is of Cardiovascular Performance, 1985 ACM Thirteenth Annual Computer Science Conference, ACM, New York.

PARISI, A.V., \& ALLEN, G.D., (1991a), A Fitness Analysis System with an Intelligent Interface, in press, Computers in Biology and Medicine.

PARISI, A.V., \& ALLEN, G.D., (1991b), Letter to the Editor: Modifications to an On-line Microcomputer Program for the Monitoring of Physiological Variables during Rest and Exercise, in press, Computers in Biology and Medicine.

PARSAYE, K., \& CHIGNELL, M., (1988), Expert Systems for Experts, John Wiley \& Sons, New York.

PATKIN, M., (1987), Ergonomics and Software, Lecture of the Year, Australian Computer Society.

PRERAU, D.S., (1990), Developing and Managing Expert Systems, Addison Wesley.

PRICE, C.J., (1990), Knowledge Engineering Toolkits, Ellis Horwood, Chichester.

RIPPLE, W.J., \& ULSHOEFER, V.S., (1987), Expert Systems and Spatial Data Models for Efficient Geographic Data Handing, Photogrammetric Engineering and Remote Sensing, Vol 53, No 10, pp 1431-1433. 
RODEWALD, L.E., (1984), BABY: An Expert system for Patient Monitoring in a Newborn Intensive Care Unit, M.S. Thesis, Computer Science Dept., University of Illinois, Champaign-Urbana.

SAINSBURY, D.A., GORE, C.J., WITHERS, R.T., ILSLEY, A.H., (1988), An On-line Microcomputer Program for the Monitoring of Physiological Variables during Rest and Exercise, Comput. Biol. Med, Vo1 18, No 1, pp 17-24.

SHAMMAS, N. C., (1986), Turbo Prolog, Byte, September 1986, pp 293295.

SHEPPARD, B., (1987), High Performance Software Analysis on the IBM PC, Byte, January 1987, pp 157-161.

SHORTLIFFE, E.H., (1976), Computer Based Medical Consultations: MYCIN, Elsevier, New York.

SHORTLIFFE, E.H., SCOTT, A.C., BISCHOFF, M.B., CAMPBELL, A.B., van MELLE, W., JACOBS, C.D., (1981), ONCOCIN: An Expert System for Oncology Protocol Management, Proceedings IJCAI-81, pp 876-881.

SIMON, B., CHABERT, A., BROWN, D., ORENSTEIN, B., (1990), Knowledge Acquisition and Verification Using Tableaux, AI'90 Workshop Proceedings.

SMITH, P., (1988), Expert System Development in Prolog and TurboProlog, Sigma Press, Wilmslow.

TANENBAUM, A. S., (1984), Structured Computer Organization, 2nd ed, Prentice Hall, New Jersey.

TOWNSEND, C., (1987), Mastering Expert Systems with Turbo Prolog, Howard W. Sams \& Co., Indianapolis.

VICKERS, J.S., KINGSTON, G.E., (1987), Modelling the Master Coach: Building an Expert System for Coaching, in Proceedings of the International Conference on Computer Assisted Learning in PostSecondary Education, Calgary, Canada.

WEITZEL, J.R., \& KERSCHBERG, L., (1989), Developing Knowledge-Based Systems: Reorganizing the System Development Life Cycle, Communications of the ACM, Vol 32, No 4, pp 482-488.

WHITE, R., (1989), Exercise Bike Wheel Revolution Detector, Project Report, School of Engineering, DDIAE. 
A P B N D I X A : 1

SY TEM COMPONENTS

Manufacturer

Microcomputer
CGA screen
132 colum dot matrix
printer
12 bit A/D converter
$\mathrm{CO}_{2}$ analyser
$\mathrm{O}_{2}$ analyser
Ventilometer
Triple port valve \&
controller
Stepper motors
Respiratory valve
Barometer
Treactmill
Meteorological
balloons
Corrugated tubing
Junction boxes
Frame for suspending
triple port valve
Vacuum pump
Ergometer
Reflective Opto switch
Circuitry

Epson

Taxan Super Vision IV

Epson

Morgan

Beckman LB-2

Beckman OM-11

Morgan

Customised

RS332-082

Hans Rudolph 2700

A.L.F.

Quinton

Totex

$3.75 \mathrm{~cm}$ diameter Fabricated at USO Fabricated at USO

Hoover 1300

Monark

RS307-913

Fabricated at use
Epson Australia Pty Ltd

Kaga Electronics Co Ltd

Epson Australia Pty Ltd

P.K. Morgan (Aust) Pty Ltd Beckman Instruments Inc Beckman Instruments Inc P.K. Morgan (Aust) Pty Ltd Danz Instrument Service

\section{Radiospares} Hans Rudolph Ine A.L. Franklin Pty Ltd Quinton Instruments Inc Totex Corporation

Hoover Aust

Monark-Crescent

Radiospares 
A P P E N I X A. 2

\section{AEROBIC TEST PROTOCOL}

1. Warm-up

(a) 10 minutes consisting of 30 seconds pedalling - 30 seconds rest. Approximate heart rate $160 \mathrm{bpm}$ (children); $150 \mathrm{bpm}$ (adults) or, for large groups

(b) 2-4 minutes pedalling with 2-3 "sprints", 4-8 secs duration each.

3-5 minutes rest between warm-up and test

2. Determine load (tension) according to body weight

3. Test - on 'go', subject pedals unloaded ergometer as fast as possible

After 2-3 seconds, apply predetermined load and start test

Test runs exactly 30 seconds

Emphasise 30 seconds - al1 out test performance is required

4. Cool down - 2-3 minutes of light pedalling immediately following test

DO NOT RETEST SAME MUSCLE GROUP WITHIN 45-60 MINUTES.

FOR TESTING LEGS THEN ARMS, 30 MINUTES REST MAY BE ADEQUATE.

FOR TESTING ARMS THEN LEGS, 20-30 MINUTES REST MAY BE ADEQUATE

\begin{tabular}{|c|c|c|c|c|}
\hline & \multicolumn{2}{|c|}{ OPTIMAL LOAD TABLE } & \multicolumn{2}{|c|}{ ( $(k P$ per $k g$ body weight) } \\
\hline & \multicolumn{2}{|r|}{ Legs } & \multicolumn{2}{|c|}{ Arms } \\
\hline & Women & Men & Women & Men \\
\hline Normal & 0.075 & $0.083-0.092$ & $0.050-0.058$ & $0.058-0.067$ \\
\hline Trained & 0.076 & $0.084-0.093$ & $0.051-0.059$ & $0.059-0.068$ \\
\hline Children (<15 years) & 0.075 & 0.075 & - & - \\
\hline Obese/very untrained & 0.074 & $0.082-0.091$ & $0.049-0.057$ & $0.057-0.066$ \\
\hline
\end{tabular}




\section{A P P ENDIX A. 3}

\section{EXAMPIE OF NORMEILE.DOC}

$\begin{array}{lllllllllll}3.0 & 153.0 & 157.33 & 158.65 & 26.75 & 25.44 & 0.95 & 5.88 & 2.2 & 27.02 & 20.48\end{array}$ $110.79504 .8 \quad 594.0 \quad 330.0 \quad 605.0 \quad 9.0 \quad 5.0 \quad 9.17 \quad 44.44 \quad 8.8$

The first thirteen values represent the means of the physiological attributes from the aerobic test. They appear in the same order in which they are stored in the file for the aerobic tests. The first value is the mean body weight, the second represents mean maximum heart rate and so on. The last eight represent the means from the anaerobic tests with the exclusion of the body weight which is not redisplayed as it already appears in the first column. 
A P PENDIX A. 4

\section{PROGRAMING THE A/D CONVERTER}

The $A / D$ converter uses eight consecutive $I / O$ address spaces starting at the base address $0710 \mathrm{H}$ or 1808 (Morgan, 1988a). These eight addresses are as follows:

$\begin{array}{lll}\text { address } & \text { location written to } & \text { location if read } \\ 1808 & \text { Timer Register 0 } & \text { Timer Register 0 } \\ 1809 & \text { Timer Register 1 } & \text { Timer Register 1 } \\ 1810 & \text { Timer Register 2 } & \text { Timer Register 2 } \\ 1811 & \text { Timer Control byte } & \\ 1812 & \text { Port B Output } & \text { LSB A/D conversion } \\ 1813 & \text { Port C Output } & \text { MSB \& status A/D } \\ 1814 & \text { A/D Channe1 select } & \text { Port A Input } \\ 1815 & \text { Timer Load Strobe } & \text { Serial Number }\end{array}$

A channel is sampled by sending the channel number to the $A / D$ channel select. Address 1813 is now read to provide the most significant byte. Bit 7 of this byte holds the status of the $A / D$ conversion. A one signifies the conversion is still in process. If this is the case, the address is read up to a maximum of 100 times until the conversion is finished. Address 1812 is then read to provide the least significant byte. Digital $I / O$ are simple read and writes to the appropriate addresses. 
A P P E D I X B

PROGRAX IISTINGS

See disc for the program source code 
FITNESS ANALYSIS SYSTEM WITH AN INTELIIGENT INTERFACE Introduction

EXFIT provides a report of generalized activities from which the coach can develop a specific training schedule to enhance an athlete's performance. The recommendations are based on the results of a series of physiological tests. The following information is a guide for using EXFIT.

The system is driven by a series of menus and screenforms. The user selects from a menu of choices displayed on the screen. Directions are always present at the bottom of the screen in the instruction window.

To run the program type EXFIT followed by <ENTER>. From now on follow the instructions at the bottom of the screen.

The user moves around each menu by using the following keys:

DOWN ARROW : move to next choice in the menu

UP ARROW : move to previous choice in the menu

PgDn : move to the last choice in the menu

PgUp : move to the first choice in the menu

Key Letter : select the choice with the key letter

The main menu presents five choices:

- Expert advice provides recommendations to the athlete to improve athletic performance.

- Athletes tests brings up a sub menu with further choices related to the running of tests on the athlete

- Comparison with means compares the athletes results with the means of the results of the other athletes in the database

- Maintain allows the user to update or maintain the data and information for providing the recommendations of the expert system.

- Online help displays some help information.

- Quit exits the system. It is also possible to exit by pressing the $<$ ESC $>$ key

The sub menu for the athletes tests option contains the following 
choices:

- Aerobic test runs the aerobic maximal oxygen uptake test

- Wingate Anaerobic test runs the Wingate test using the cycle ergometer.

- Performance uses the records on the athletes computer file to plot the athletes performance in a particular area as a function of the date of the test.

- Test report allows the printing of an athletes past or present test results.

- Display data record allows the display of the records in the athletes file. Also if required allows the deletion of unwanted or incorrect records

- Exit exits back to main menu. Pressing the <ESC> key has the same effect.

The above options are described in greater detail below.

\section{Expert Advice}

- On selection of this option there is disc activity for several seconds while the data and information files are loaded into RAM. The expert system consults these files to provide the recommendations. The files are called DATA.DOC and INFORMATION.DOC and must exist on the disc in drive $B$ or $C$.

- Select the mode of output. The choices are to display on the screen, a printout or both.

- Select the analysis to be carried out on the athlete. The analyses available are stored in the data and information files and are displayed in a menu.

- The tests to be carried out for each analysis are also stored in the data and information files. The user selects the result obtained by the athlete in each test.

- On entry of all the results for the tests, the data and information files are consulted to conclude one or more diagnoses. Having obtained the diagnoses, EXFIT again searches the data and information to obtain one or more aims with the associated activities for the given diagnoses. For each activity EXFIT performs another search to find any explanations, warnings and follow up procedures.

- If the display fills more than one screen press <PgDn> or <PgUp> for the next or previous screens.

- On completion of the display press <ESC> to return to the EXFIT 
menu.

\section{Comparison with means}

- Enter the athletes filename or press <ENTER> and use the arrow keys to select the file from the displayed directory.

- Select the variables to be compared. Press $\langle F 10\rangle$ once selection is complete. A maximum of 21 variables may be compared at a time.

- The user has the option of the program recalculating the means for the group of athletes before any comparison is made. Answer $Y$ or $N$ as required.

- If the user answers $Y$ the program will scan all files on the disc with an extension of .DAT. For each file the filename and athletes age are displayed and the user is requested whether the particular file is to be used in calculating the means. Answer $\mathrm{Y}$ or $\mathrm{N}$ as appropriate and the process is repeated for the next file.

- Once all the .DAT files on the disc have been scanned the user is asked if it necessary to repeat for the next disc. This is required if there are more files to be used on other discs. Answer $Y$ or $N$ as appropriate. On completion of the scan of all the .DAT files, the calculated means are displayed with the option of pressing $\langle E S C\rangle$ to exit at this point or pressing any other key to continue.

- Pressing any other key will repeat the process for the next test. Once calculated the means are recorded on a disc file called NORMFILE.DOC.

- The means for the variables selected are displayed and again the user can select to exit or to continue.

- The comparisons are now displayed on the screen. The means for the group are indicated as $0 \%$ with the athlete's results plotted relative to this up to 208 either side. If the difference is larger than 208, a ' $*$ ' is plotted at the appropriate extremity. The athletes result for each variable is written on the lefthand side of the graph.

- Press <F10> to obtain a printout of the graph or press any other key to exit without printing.

\section{Maintain}

- This option requires the files Maintain.exe, Data.doc and Information.doc to exist on the disc in drive $B$ or $C$.

- Use the line menu at the bottom of the screen to load the data and information files.

- Maintain uses two screens. The first screen allows the updating or 
modification of the analyses, tests and test results. The second screen keeps these three fixed and allows updating or modification to the remainder.

- Remember to save the files if you have made any changes that you wish to save.

\section{Online help}

- Provides generalized help information. Context sensitive help is also provided at the more critical stages of the dialogue. This is available by pressing the $\langle F 1>$ key. The arrow keys, $\langle$ Pgup $\rangle$ and $\langle\mathrm{PgDn}\rangle$ allow movement around the help windows. Pressing $<E S C\rangle$ exits the help window and returns to the menu.

\section{Aerobic and wingate Anaerobic test}

\section{Completion of form}

- Once each field is completed move to the next field by pressing <ENTER> or using the arrow keys. If no input is required for a field or if a field is to remain unchanged press <ENTER> without typing anything.

- Ensure information is entered in the SURNAME, GIVEN NAME fields. The program uses the surname and first initial and the extension .DAT to generate the filename for the athlete. Ensure the date is correct as this is used in date stamping the test record that is written to the athletes file.

- Within a field, use the $->$ and <- arrow keys to move over previously entered text in a field. The $\langle$ DEL $\rangle$ key will delete any unwanted characters.

- Ensure all the information is correct before pressing $\langle F 10\rangle$ to continue.

- Select either automatic or manual data acquisition

- The next form requires data related to the analysis. Enter the data requested using a decimal point as required. Use the arrow keys and <ENTER> to move around the form.

\section{Aerobic Test - Automatic Data Acquisition}

- At the user specified time interval, the triple port valve rotates and the athlete's expired air is collected in the next balloon. Note that this rotation takes place only when the athlete has completed an inspiration. While the rotation is taking place the software samples the ventilometer to read the volume of inspired air and the number of inspirations. The software waits half a time interval before sampling the gas analysers. This ensures the gas analysers have stabilised before being sampled. 
- Once sampling is complete for the time interval, the software performs the calculations. The results are printed on a printer if one is on-line. The results are also displayed on the screen or if requested $\mathrm{VO}_{2}$ and $\mathrm{RER}$ are plotted as a function of time.

- Press <ESC> to end the test at the end of the next time interval. The results calculated for each time interval are now displayed on the screen. The user selects the record(s) to be used in storing to the athletes file. If more than one record is selected the software calculates the average of each variable in the records selected and the averages are stored to the file.

\section{Wingate Anaerobic Test - Automatic Data Acquisition}

- While the athlete pedals the unloaded ergometer as fast as possible, strike any key to select the 'gearing'. The program measures the rate of detection of contrasting spots on the wheel and instructs the circuitry to look at every spot or every second spot or every fourth spot or every eight spot according to the speed of revolution of the wheel. The gearing selected is shown on the display on the junction box. Once selected the gearing remains constant for the duration of the test. In this manner the equipment can be used to test subjects over the complete range of capabilities.

- After 2-3 seconds the predetermined load is applied and press any key to start counting. At the end of the specified time interval the computer beeps and the information on the total pedal revolutions and the maximum and minimum revolutions in a five second period are displayed. At this point the user has the option of pressing $\angle E S C>$ to exit or pressing any other key to continue with the calculations and data recording.

- The calculated variables are displayed on the screen. Press <ESC> to exit without recording the information on the athletes file. Pressing any other key will record the information on the file and will display the print menu.

\section{Performance}

The results for the athlete may be plotted as a function of the date of the test. Ensure the data disc is in the drive and:

- Enter the athlete's filename or press <ENTER> and use the arrow keys to select the file from the displayed directory.

- Select the test required and the variable to be plotted.

- Press $\langle F 10\rangle$ to obtain a printout of the graph or press any other key to exit without printing. 


\section{Test report}

- Enter the athlete's filename or press <ENTER> and use the arrow keys to select the file from the displayed directory.

- Select the test required and the option of no printout, print latest results only, print all previous results or specify the number of past results to print.

- Ensure that a printer is connected and on line.

\section{Display data record}

- Enter the athlete's filename or press <ENTER> and use the arrow keys to select the file from the displayed directory.

- Select the test required. The records are now displayed on the screen. Use the arrow keys, $\langle\mathrm{PgDn}\rangle,\langle\mathrm{PgUp}\rangle,\langle\mathrm{Home}\rangle$ and $\langle$ End $\rangle$ keys to move around the screen. Press <ESC> to exit from the screen. The user is now allowed the option of deleting any unwanted records. If the user selects this option, the user selects the records to be deleted from a menu. Press $\langle F 10\rangle$ once selection is complete. For each record selected, the user is prompted if it is OK to delete. Enter $\mathrm{Y}$ or $\mathrm{N}$ as appropriate. 


\section{A P PENDI D}

\section{SAMPIE RECOMNENDATIONS}

RECOMOMENDATION NO. 1

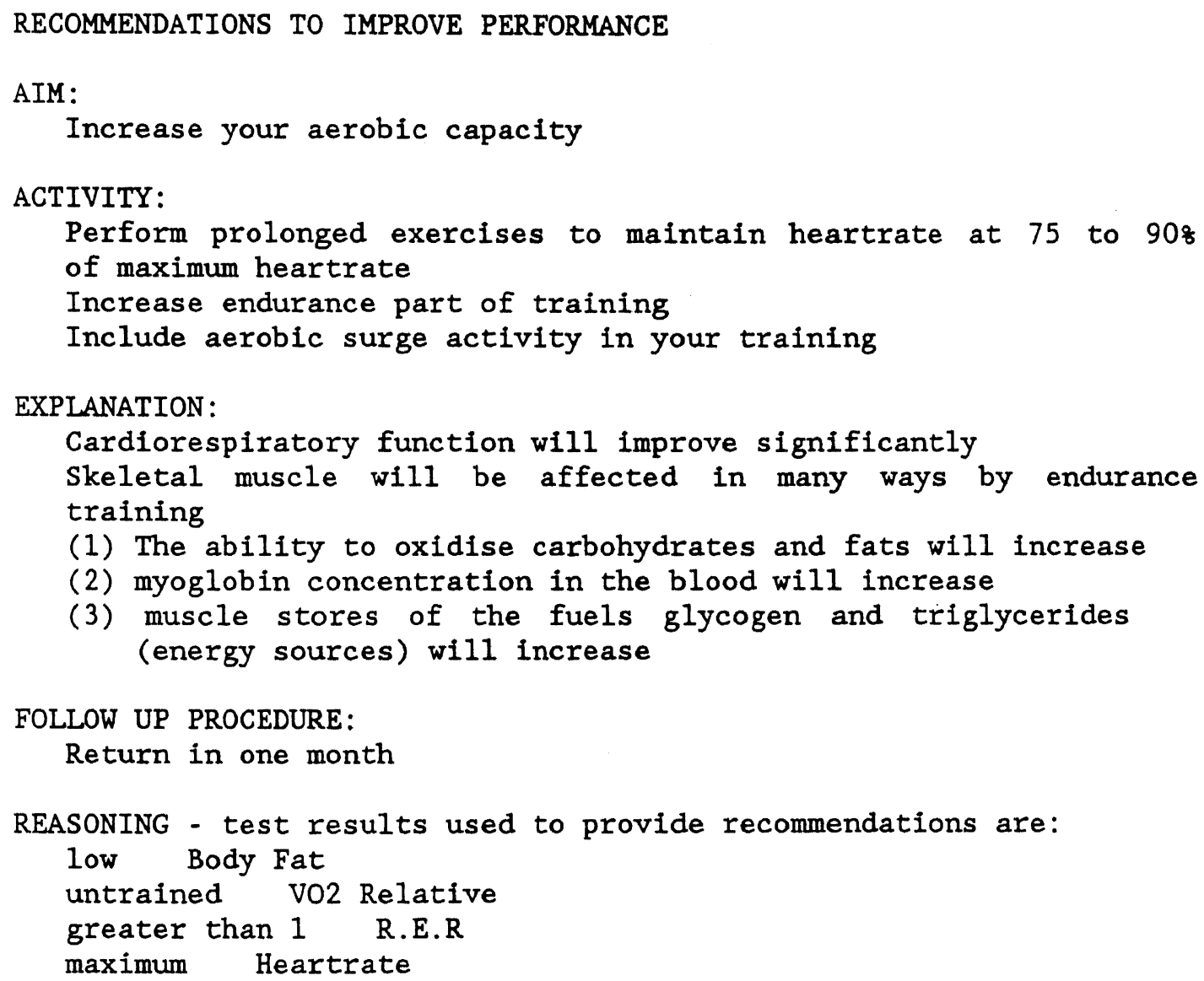


RECOMGENDATION NO. 2

RECOMMENDATIONS TO IMPROVE PERFORMANCE

AIM :

Increase your total anaerobic power output

ACTIVITY :

Overload your muscles by anaerobic exercise against resistances which exceed those normally encountered

Use intensive intervals of varying 20 to 40 second surges during normal training

Perform weight training with a view to increasing anaerobic power output

WARNING :

In weight training programmes the overload must be progressive throughout the training period

In any weight training session, larger muscle groups should be exercised before smaller groups

For resistance training consecutive exercises should not involve the same muscle groups

FOLLOW UP PROCEDURE:

Return in one month

REASONING - test results used to provide recommendations are:

low Body Fat

untrained Total Power 


\section{RECOMGENDATION NO. 3}

RECOMMENDATIONS TO IMPROVE PERFORMANCE

AIM:

Reduce your body fat

ACTIVITY:

Increase aerobic energy expenditure

Check dietary intake over a week and determine dietary intake relative to energy expenditure and adjust as required

Progressively increase aerobic training

EXPLANATION:

The weight loss from this activity is primarily in the form of fat weight

Excess expenditure of $3500 \mathrm{kcal}$ is required to $10 \mathrm{se} 0.45 \mathrm{~kg}$ of body fat

By manipulation of diet and exercise create an imbalance in the energy equation which promotes fat loss until the desired fat level is obtained

WARNING :

Rate of sustained weight loss should not exceed $0.45 \mathrm{~kg} /$ week without professional supervision

FOLLOW UP PROCEDURE:

Record the weekly body weight (taken at the same time on a given day)

Return in six weeks

REASONING - test results used to provide recommendations are:

high Body Fat

trained Peak Power 


\section{RECOMMENDATION NO. 4}

\section{RECOMMENDATIONS TO IMPROVE PERFORMANCE}

AIM:

Increase glycogen level

Increase the level of aerobic markers in muscle

ACTIVITY:

Increase endurance training (intensity and duration)

Increase carbohydrate content of diet

Include 60 to 240 second surges of exercise in an endurance training programme

\section{EXPLANATION:}

An increase in the stores of muscle glycogen can improve endurance performance. Muscle glycogen is a primary source of energy during prolonged aerobic activity

Cardiorespiratory function will improve significantly

Skeletal muscle will be affected in many ways by endurance training

(1) The ability to oxidise carbohydrates and fats will increase

(2) myoglobin concentration in the blood will increase

(3) muscle stores of the fuels glycogen and triglycerides (energy sources) will increase

WARNING :

Do not increase your overall caloric load

FOLLOW UP PROCEDURE:

Return in six weeks

REASONING - test results used to provide recommendations are:

normal Glycogen Level

moderately trained Aer Markers - SDH, CS, CYT-OX 


\section{RECOMOENDATION NO. 5}

\section{RECOMMENDATIONS TO IMPROVE PERFORMANCE}

AIM :

Increase the glycogen level in muscle

Increase the level of anaerobic markers in muscle

\section{ACTIVITY:}

Increase carbohydrate content of diet

Intensive anaerobic surges ranging from 20 to 60 seconds duration Increase weight training load

EXPLANATION:

An increase in the stores of muscle glycogen can enhance repetitive anaerobic performance

Anaerobic capacity can be increased only by specific anaerobic training

WARNING :

Do not increase your overall caloric load

Increase anaerobic training load gradually

FOLLOW UP PROCEDURE:

Return in six weeks

REASONING - test results used to provide recommendations are:

mod. high Glycogen Level

untrained Ana Markers - PFK, PHOS 
RECOMOMENDATION NO. 6

\section{RECOMMENDATIONS TO IMPROVE PERFORMANCE}

AIM :

Increase your Hemoglobin concentration

Increase your Iron level

Increase your Ferritin level

Increase your red blood count

ACTIVITY:

Increase iron intake

Consult your sports medical practitioner for advice

Iron supplements may be recommended by your sports medical practitioner

EXPLANATION:

The greatest portion of oxygen is carried by the red blood cells in combination with the iron component of hemoglobin

Lean red meat is a prime dietary source of iron

Other food sources of iron are liver, heart, kidney, egg yolk, dried beans, nuts, prune juice, wheat germ

WARNING :

Overdoses of iron supplements can be toxic; professional supervision is advisable

FOLLOW UP PROCEDURE:

Return if an unexplained drop off in endurance performance occurs

Return in 4 to 6 weeks

REASONING - test results used to provide recommendations are:

low Hemoglobin Conc.

medium Iron Level

medium Ferritin Level

low Red Blood Count 
RECOMUENDATION NO. 7

RECOMMENDATIONS TO IMPROVE PERFORMANCE

AIM:

Increase max. blood lactate level after max. aerobic/anaerobic performance

ACTIVITY:

Increase intensive aerobic and anaerobic training

EXPLANATION :

Prolonged and intensive (20 to 60 seconds) anaerobic training will increase the capacity of the lactic acid mechanism. The bursts may be incorporated into the aerobic training component of the programme

FOLLOW UP PROCEDURE:

Return in six weeks

REASONING - test results used to provide recommendations are: untrained Max Blood Lactate Level 


\section{RECOMAENDATION NO. 8}

\section{RECOMMENDATIONS TO IMPROVE PERFORMANCE}

AIM :

Increase your anaerobic threshold

ACTIVITY:

Progressively increase intensive endurance training (Maintain exercise heartrate at 75 to 908 of maximum heartrate)

EXPLANATION :

The workload at which OBLA occurs increases with intense aerobic training. This is due to the training effect which enhances the body's ability to use aerobic processes during exercise before anaerobic processes contribute significantly

FOLLOW UP PROCEDURE:

Return in six weeks

REASONING - test results used to provide recommendations are: no change with training OBLA 